\title{
Numerical Analysis of a Rotor Dynamics in the Magneto-Hydrodynamic Field
}

\author{
Jan Awrejcewicz ${ }^{1}$ and Larisa P. Dzyubak ${ }^{2}$ \\ ${ }^{1}$ Technical University of Łódź \\ 2National Technical University "Kharkov Polytechnic Institute" \\ 1Poland \\ 2Ukraine
}

\section{Introduction}

In general, rotating machinery elements are frequently met in mechanical/mechatronical engineering, and in many cases their non-linear dynamics causes many harmful effects, i.e. noise and vibrations. In particular, nonlinear rotordynamics plays a crucial role in understanding various nonlinear phenomena and in spite of its long research history (see for instance (Tondl, 1965; Someya, 1998; Rao, 1991; Gasch et al., 2002; Muszyńska, 2005) and the references therein) it still attracts attention of many researchers and engineers. Since the topics related to nonlinear rotordynamics are broadband and cover many interesting aspects related to both theory and practice, in this chapter we are aimed only on analysis of some problems related to rotor suspended in a magneto-hydrodynamics field in the case of soft and rigid magnetic materials.

The magnetic, magneto-hydrodynamic and also piezoelectric bearings are used in many mechanical engineering applications in order to support a high-speed rotor, provide vibration control, to keep lower rotating friction losses and to potentially avoid flutter instability. There are a lot of publications devoted to the dynamics analysis and control of a rotor supported on various bearings systems. The conditions for active close/open-loop control of a rigid rotor supported on hydrodynamic bearings and subjected to harmonic kinematical excitation are presented in (Kurnik, 1995; Dziedzic \& Kurnik, 2002). The methodology for modeling lubricated revolute joints in constrained rigid multibody systems is described in (Flores et al., 2009). The hydrodynamic forces, used in the dynamic analysis of journal-bearings, which include both squeeze and wedge effects, are evaluated from the system state variables and included into the equations of motion of the multibody system. To analyze the dynamic behavior of rub-impact rotor supported by turbulent journal bearings and lubricated with couple stress fluid under quadratic damping the authors of (Chang-Jian \& Chen, 2009) have used the system state trajectory, Poincaré maps, power spectrum, bifurcation diagrams and Lyapunov exponents. It was detected the dynamic motion as periodic, quasi-periodic and chaotic types.

In (Zhang \& Zhan, 2005; Li et al., 2006) a rotor-active magnetic bearings (rotor-AMB) systems with time-varying stiffness are considered. Using the method of multiple scales a governing nonlinear equation of motion for the rotor-AMB system with 1-dof is transformed to the averaged equation and then the bifurcation theory and the method of detection function are used to analyze the bifurcations of multiple limit cycles of the averaged equation. 


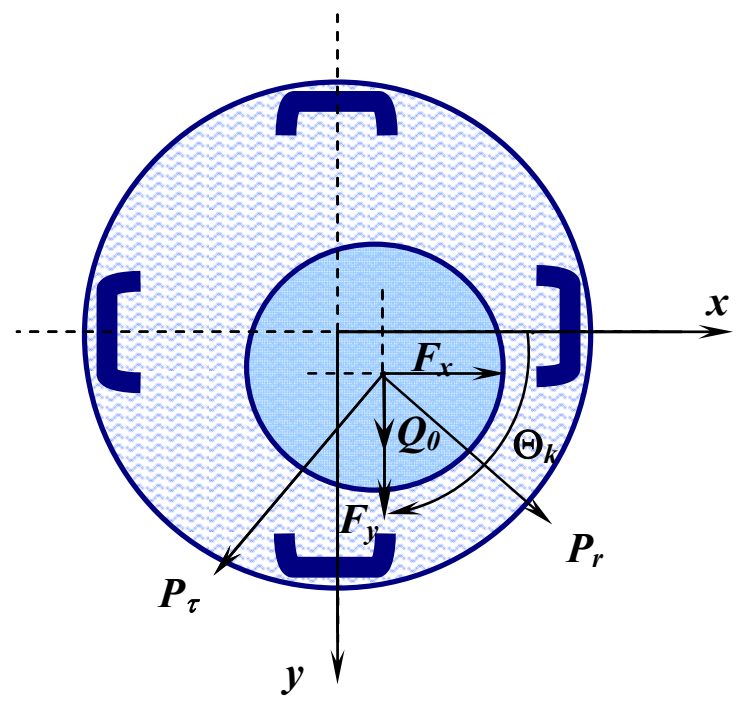

Fig. 1. The cross-section diagram of a rotor symmetrically supported on the magnetohydrodynamic bearing

In the present chapter 2-dof nonlinear dynamics of the rotor supported on the magnetohydrodynamic bearing (MHDB) system is analyzed in the cases of soft and rigid magnetic materials. In the case of soft magnetic materials the analytical solutions have been obtained by means of the method of multiple scales (Nayfeh \& Mook, 2004). Rigid magnetic materials possess hysteretic properties which are realized in the frames of the present work by means of Bouc-Wen hysteretic model. This model allows simulating hysteretic loops of various forms for systems from very different fields (Awrejcewicz \& Dzyubak, 2007). Chaotic regions and the amplitude level contours of the rotor vibrations have been obtained in various control parameter planes.

\section{Mathematical model of the rotor suspended in the magneto-hydrodynamic field}

Consider a uniform symmetric rigid rotor (Fig. 1) which is supported by a magnetohydrodynamic bearing system. The four-pole legs are symmetrically placed in the stator. $F_{\mathrm{k}}$ is the electromagnetic force produced by the kth opposed pair of electromagnet coils. This force is controlled by electric currents

$$
i_{k}=i_{0} \pm \Delta i_{k}
$$

can be expressed in the form

$$
F_{k}=-\frac{2 \mu_{0} A N^{2} i_{0}}{\left(2 \delta+l / \mu^{*}\right)^{2}} \Delta i_{k}
$$


where $i_{0}$ denotes bias current in the actuators electric circuits, $\mu_{0}$ is the magnetic permeability of vacuum, $A$ is the core cross-section area, $N$ is the number of windings of the electromagnet, $\delta$ is the air gap in the central position of the rotor with reference to the bearing sleeve, $l$ is the total length of the magnetic path, the constant value $\mu^{*}=B_{s} /\left(\mu_{0} H_{s}\right)$ denotes the magnetic permeability of the core material; the values of the magnetic induction $B_{s}$ and magnetizing force $H_{s}$ define the magnetic saturation level. $\theta_{k}$ is the angle between axis $x$ and the kth magnetic actuator. $Q_{0}$ is the vertical rotor load identified with its weight, $\left(P_{r}, P_{\tau}\right)$ are the radial and tangential components of the dynamic oil-film action, respectively. Equations of motion of the rotor are represented in the following form (Kurnik, 1995; Dziedzic \& Kurnik, 2002; Osinski, 1998)

$$
\begin{gathered}
m^{*} \ddot{x}^{*}=P_{r}{ }^{*}\left(\rho, \dot{\rho}^{*}, \dot{\varphi}^{*}\right) \cos \varphi-P_{\tau}^{*}\left(\rho, \dot{\varphi}^{*}\right) \sin \varphi+\sum_{k=1}^{K}{F_{k}}^{*} \cos \theta_{k}+Q_{x}{ }^{*}(t), \\
m^{*} \ddot{y}^{*}=P_{r}^{*}\left(\rho, \dot{\rho}^{*}, \dot{\varphi}^{*}\right) \sin \varphi+P_{\tau}^{*}\left(\rho, \dot{\varphi}^{*}\right) \cos \varphi+\sum_{k=1}^{K} F_{k}^{*} \sin \theta_{k}+Q_{0}{ }^{*}+Q_{y}{ }^{*}(t), \\
P_{r}^{*}\left(\rho, \dot{\rho}^{*}, \dot{\varphi}^{*}\right)=-2 C^{*}\left\{\frac{\rho^{2}\left(\omega^{*}-2 \dot{\varphi}^{*}\right)}{p(\rho) q(\rho)}+\frac{\rho \dot{\rho}^{*}}{p(\rho)}+\frac{2 \dot{\rho}^{*}}{\sqrt{p(\rho)}} \operatorname{arctg} \sqrt{\frac{1+\rho}{1-\rho}}\right\}, \\
P_{\tau}^{*}\left(\rho, \dot{\varphi}^{*}\right)=\pi C^{*} \frac{\rho\left(\omega^{*}-2 \dot{\varphi}^{*}\right)}{q(\rho) \sqrt{p(\rho)}} .
\end{gathered}
$$

Here $m^{*}$ denotes the rigid rotor mass, $\left(x^{*}, y^{*}\right)$ are the Cartesian coordinates of the rotor center; $Q_{x}{ }^{*}(t), Q_{y}{ }^{*}(t)$ are the external excitation characterizing bearing housing movements. We are considering vibrations of the rotor excited by harmonic movements of the bearing foundation in the vertical direction

$$
Q_{x}^{*}(t)=0, Q_{y}^{*}(t)=Q^{*} \sin \Omega^{*} t^{*},
$$

where $Q^{*}$ and $\Omega^{*}$ are the amplitude and frequency of the external excitation, respectively. Constant $C^{*}$ is defined as

$$
C^{*}=\frac{6 \mu_{s} R_{c} L_{c}}{\delta_{s}^{2}}
$$

Parameters $\mu_{\mathrm{s}}, \delta_{\mathrm{s}}, R_{\mathrm{c}}, L_{\mathrm{c}}$ denote oil viscosity, relative bearing clearance, journal radius and total bearing length, respectively. $(\rho, \phi)$ are the polar coordinates, $p(\rho)=1-\rho^{2}$, $q(\rho)=2+\rho^{2}$ are the functions conditional $\rho$.

To represent the equations of motion in a dimensionless form the following changes of variables and parameters are introduced:

$$
t=\omega^{*} t^{*}, \dot{\varphi}=\frac{\dot{\varphi}^{*}}{\omega^{*}}, \dot{\rho}=\frac{\dot{\rho}^{*}}{\omega^{*}}, x=\frac{x^{*}}{c^{*}}, \dot{x}=\frac{\dot{x}^{*}}{\omega^{*} c^{*}}, \ddot{x}=\frac{\ddot{x}^{*}}{\omega^{* 2} c^{*}}, y=\frac{y^{*}}{c^{*}}, \dot{y}=\frac{\dot{y}^{*}}{\omega^{*} c^{*}},
$$




$$
\begin{gathered}
\ddot{y}=\frac{\ddot{y}^{*}}{\omega^{* 2} c^{*}}, C=\frac{C^{*}}{m^{*} \omega^{*} c^{*}}, \Omega=\frac{\Omega^{*}}{\omega^{*}}, Q=\frac{Q^{*}}{m^{*} \omega^{* 2} c^{*}}, Q_{0}=\frac{Q_{0}^{*}}{m^{*} \omega^{* 2} c^{*}}, \\
F_{k}=\frac{F_{k}^{*}}{m^{*} \omega^{* 2} c^{*}}, P_{r}=\frac{P_{r}^{*}}{m^{*} \omega^{* 2} c^{*}}, P_{\tau}=\frac{P_{\tau}^{*}}{m^{*} \omega^{* 2} c^{*}},
\end{gathered}
$$

where $\omega^{*}$ is the rotation speed of the rotor; $c^{*}$ is the bearing clearance.

Thus the dimensionless equations of motion take the form

$$
\begin{gathered}
\ddot{x}=P_{r}(\rho, \dot{\rho}, \dot{\varphi}) \cos \varphi-P_{\tau}(\rho, \dot{\varphi}) \sin \varphi+F_{x}, \\
\ddot{y}=P_{r}(\rho, \dot{\rho}, \dot{\varphi}) \sin \varphi+P_{\tau}(\rho, \dot{\varphi}) \cos \varphi+F_{y}+Q_{0}+Q \sin \Omega t, \\
P_{r}(\rho, \dot{\rho}, \dot{\varphi})=-2 C\left\{\frac{\rho^{2}(1-2 \dot{\varphi})}{p(\rho) q(\rho)}+\frac{\rho \dot{\rho}}{p(\rho)}+\frac{2 \dot{\rho}}{\sqrt{p(\rho)}} \operatorname{arctg} \sqrt{\frac{1+\rho}{1-\rho}}\right\}, P_{\tau}(\rho, \dot{\varphi})=\pi C \frac{\rho(1-2 \dot{\varphi})}{q(\rho) \sqrt{p(\rho)}} .
\end{gathered}
$$

Here

$$
\begin{gathered}
x=\rho \cos \varphi, y=\rho \sin \varphi, \dot{\varphi}=\frac{\dot{y} x-\dot{x} y}{\rho^{2}}, \dot{\rho}=\frac{x \dot{x}+y \dot{y}}{\rho}, \rho=\sqrt{x^{2}+y^{2}}, \\
\cos \varphi=\frac{x}{\sqrt{x^{2}+y^{2}}}, \sin \varphi=\frac{y}{\sqrt{x^{2}+y^{2}}} ;
\end{gathered}
$$

the magnetic control forces are expressed as follows

$$
F_{x}=-\gamma \dot{x}-\lambda\left(x-x_{0}\right), F_{y}=-\gamma \dot{y}-\lambda\left(y-y_{0}\right),
$$

where $\left(x_{0}, y_{0}\right)$ are the coordinates of the rotor static equilibrium, $\gamma$ and $\lambda$ are the control parameters.

\section{Soft magnetic materials}

In this section, we consider 2-dof dynamics of the rotor in the MHDB system without taking hysteresis into account.

\subsection{The non-resonant case}

The right-hand sides of Eqs (1) were expanded in Taylor's series and the origin was shifted to the location of the static equilibrium $\left(x_{0}, y_{0}\right)$ for the convenience of the investigation. The linear and quadratic terms were kept. So, the reformed equations of motion are as follows:

$$
\begin{aligned}
& \ddot{x}+\alpha x-\beta \dot{y}=-2 \hat{\mu}_{1} \dot{x}+\alpha_{1} x^{2}+\alpha_{2} y^{2}+\alpha_{3} x \dot{x}+\alpha_{4} x y+\alpha_{5} x \dot{y}+\alpha_{6} \dot{x} y+\alpha_{7} y \dot{y}, \\
& \ddot{y}+\alpha y+\beta \dot{x}=-2 \hat{\mu}_{2} \dot{y}+\beta_{1} x^{2}+\beta_{2} y^{2}+\beta_{3} x \dot{x}+\beta_{4} x y+\beta_{5} x \dot{y}+\beta_{6} \dot{x} y+\beta_{7} y \dot{y}+F \cos (\Omega t+\tau) .
\end{aligned}
$$

We seek the first-order solution for small but finite amplitudes in the form 


$$
\begin{aligned}
& x=\varepsilon x_{1}\left(T_{0}, T_{1}\right)+\varepsilon^{2} x_{2}\left(T_{0}, T_{1}\right)+\ldots, \\
& y=\varepsilon y_{1}\left(T_{0}, T_{1}\right)+\varepsilon^{2} y_{2}\left(T_{0}, T_{1}\right)+\ldots,
\end{aligned}
$$

where $\varepsilon$ is the small, dimensionless parameter related to the amplitudes and $T_{n}=\varepsilon^{n} t$ $(n=0,1)$ are the independent variables. It follows that the derivatives with respect to $t$ become expansions in terms of the partial derivatives with respect to $T_{\mathrm{n}}$ according to

$$
\begin{gathered}
\frac{d}{d t}=\frac{\partial}{\partial T_{0}} \frac{\partial T_{0}}{\partial t}+\frac{\partial}{\partial T_{1}} \frac{\partial T_{1}}{\partial t}+\frac{\partial}{\partial T_{2}} \frac{\partial T_{2}}{\partial t}+\ldots=D_{0}+\varepsilon D_{1}+\varepsilon^{2} D_{2}+\ldots \\
\frac{d^{2}}{d t^{2}}=\left(D_{0}+\varepsilon D_{1}+\varepsilon^{2} D_{2}+\ldots\right)^{2}=D_{0}^{2}+2 \varepsilon D_{0} D_{1}+\varepsilon^{2}\left(D_{1}^{2}+2 D_{0} D_{2}\right)+\ldots, \text { where } D_{k}=\frac{\partial}{\partial T_{k}} .
\end{gathered}
$$

To analyze the non-resonant case the forcing term is ordered so that it appears at order $\varepsilon$. Thus, we recall in (2) $F=\varepsilon f, \hat{\mu}_{n}=\varepsilon \mu_{n}$. Substituting (3) into (2) and equating coefficients of similar powers of $\varepsilon$ we obtain

Order $\varepsilon$

$$
\begin{aligned}
& D_{0}^{2} x_{1}+\alpha x_{1}-\beta D_{0} y_{1}=0 \\
& D_{0}^{2} y_{1}+\alpha y_{1}+\beta D_{0} x_{1}=f \cos \left(\Omega T_{0}+\tau\right)
\end{aligned}
$$

Order $\varepsilon^{2}$

$$
\begin{aligned}
& D_{0}^{2} x_{2}+\alpha x_{2}-\beta D_{0} y_{2}=-2 D_{0}\left(D_{1} x_{1}+\mu_{1} x_{1}\right)+\beta D_{1} y_{1}+\alpha_{1} x_{1}^{2}+\alpha_{2} y_{1}^{2}+ \\
& \alpha_{3} x_{1} D_{0} x_{1}+\alpha_{4} x_{1} y_{1}+\alpha_{5} x_{1} D_{0} y_{1}+\alpha_{6} y_{1} D_{0} x_{1}+\alpha_{7} y_{1} D_{0} y_{1} \\
& D_{0}^{2} y_{2}+\alpha y_{2}+\beta D_{0} x_{2}=-2 D_{0}\left(D_{1} y_{1}+\mu_{2} y_{1}\right)-\beta D_{1} x_{1}+\beta_{1} x_{1}^{2}+\beta_{2} y_{1}^{2}+ \\
& \beta_{3} x_{1} D_{0} x_{1}+\beta_{4} x_{1} y_{1}+\beta_{5} x_{1} D_{0} y_{1}+\beta_{6} y_{1} D_{0} x_{1}+\beta_{7} y_{1} D_{0} y_{1} .
\end{aligned}
$$

The solution of (4) is expressed in the form

$$
\begin{aligned}
& x_{1}=A_{1}\left(T_{1}\right) \exp \left(i \omega_{1} T_{0}\right)+A_{2}\left(T_{1}\right) \exp \left(i \omega_{2} T_{0}\right)+\Phi_{1} \exp \left[i\left(\Omega T_{0}+\tau\right)\right]+C C, \\
& y_{1}=\Lambda_{1} A_{1}\left(T_{1}\right) \exp \left(i \omega_{1} T_{0}\right)+\Lambda_{2} A_{2}\left(T_{1}\right) \exp \left(i \omega_{2} T_{0}\right)+\Phi_{2} \exp \left[i\left(\Omega T_{0}+\tau\right)\right]+C C,
\end{aligned}
$$

where CC denotes the complex conjugate of the preceding terms, $A_{1}$ and $A_{2}$ are the arbitrary functions of $T_{1}$ at this level of approximation,

$$
\Lambda_{n}=\frac{\omega_{n}^{2}-\alpha}{\omega_{n} \beta} i, \Phi_{1}=\frac{i}{2} \frac{\beta \Omega f}{\left(\alpha-\Omega^{2}\right)^{2}-\beta^{2} \Omega^{2}}, \Phi_{2}=\frac{1}{2} \frac{f\left(\alpha-\Omega^{2}\right)}{\left(\alpha-\Omega^{2}\right)^{2}-\beta^{2} \Omega^{2}},(\mathrm{n}=1,2) .
$$

$\omega_{\mathrm{n}}$ are assumed to be distinct and $\omega_{\mathrm{n}}^{2}$ are the roots of the characteristic equation

$$
\operatorname{det}\left(\begin{array}{cccc}
-\lambda & 1 & 0 & 0 \\
-\alpha & -\lambda & 0 & \beta \\
0 & 0 & -\lambda & 1 \\
0 & -\beta & -\alpha & -\lambda
\end{array}\right)=\lambda^{4}+\left(2 \alpha+\beta^{2}\right) \lambda^{2}+\alpha^{2}=\omega_{n}^{4}-\left(2 \alpha+\beta^{2}\right) \omega_{n}^{2}+\alpha^{2}=0
$$




$$
\begin{gathered}
\lambda_{1,2}= \pm i \omega_{1}, \lambda_{3,4}= \pm i \omega_{2}, \lambda_{1,2}= \pm \frac{1}{2} \sqrt{-4 \alpha-2 \beta^{2}+2 \beta \sqrt{\beta^{2}+4 \alpha}}, \\
\lambda_{3,4}= \pm \frac{1}{2} \sqrt{-4 \alpha-2 \beta^{2}-2 \beta \sqrt{\beta^{2}+4 \alpha}} .
\end{gathered}
$$

Substitution of (6) into (5) gives

$$
\begin{aligned}
& D_{0}^{2} x_{2}+\alpha x_{2}-\beta D_{0} y_{2}=\left[-2 i \omega_{1}\left(A_{1}^{\prime}+\mu_{1} A_{1}\right)+\beta \Lambda_{1} A_{1}^{\prime}\right] \exp \left(i \omega_{1} T_{0}\right)+ \\
& {\left[-2 i \omega_{2}\left(A_{2}^{\prime}+\mu_{1} A_{2}\right)+\beta \Lambda_{2} A_{2}^{\prime}\right] \exp \left(i \omega_{2} T_{0}\right)+\ldots+C C,} \\
& D_{0}^{2} y_{2}+\alpha y_{2}+\beta D_{0} x_{2}=\left[-2 i \omega_{1} \Lambda_{1}\left(A_{1}^{\prime}+\mu_{2} A_{1}\right)-\beta A_{1}^{\prime}\right] \exp \left(i \omega_{1} T_{0}\right)+ \\
& {\left[-2 i \omega_{2} \Lambda_{2}\left(A_{2}^{\prime}+\mu_{2} A_{2}\right)-\beta A_{2}^{\prime}\right] \exp \left(i \omega_{2} T_{0}\right)+\ldots+C C .}
\end{aligned}
$$

The terms, which do not influence solvability conditions, are not presented in the last equations and replaced by dots.

To determine the solvability conditions of (8), following to the method of undetermined coefficients we seek a particular solution in the form

$$
\begin{aligned}
& x_{2}=P_{11} \exp \left(i \omega_{1} T_{0}\right)+P_{12} \exp \left(i \omega_{2} T_{0}\right), \\
& y_{2}=P_{21} \exp \left(i \omega_{1} T_{0}\right)+P_{22} \exp \left(i \omega_{2} T_{0}\right)
\end{aligned}
$$

with unknowns $P_{11}, P_{12}, P_{21}$ and $P_{22}$. Substitution of expressions (9) into (8) and collection of coefficients at $\exp \left(i \omega_{1} T_{0}\right)$ and $\exp \left(i \omega_{2} T_{0}\right)$ yields

$$
\begin{gathered}
\left(\alpha-\omega_{n}^{2}\right) P_{1 n}-i \beta \omega_{n} P_{2 n}=R_{1 n}, \\
i \beta \omega_{n} P_{1 n}+\left(\alpha-\omega_{n}^{2}\right) P_{2 n}=R_{2 n} \quad(\mathrm{n}=1,2),
\end{gathered}
$$

where

$$
\begin{array}{ll}
R_{11}=-2 i \omega_{1}\left(A_{1}^{\prime}+\mu_{1} A_{1}\right)+\beta \Lambda_{1} A_{1}^{\prime}, & R_{12}=-2 i \omega_{2}\left(A_{2}^{\prime}+\mu_{1} A_{2}\right)+\beta \Lambda_{2} A_{2}^{\prime}, \\
R_{21}=-2 i \omega_{1} \Lambda_{1}\left(A_{1}^{\prime}+\mu_{2} A_{1}\right)-\beta A_{1}^{\prime}, & R_{22}=-2 i \omega_{2} \Lambda_{2}\left(A_{2}^{\prime}+\mu_{2} A_{2}\right)-\beta A_{2}^{\prime} .
\end{array}
$$

Taking into account the characteristic equation (7), the determinant $\Delta$ of the set of linear algebraic equations relative to $P_{1 \mathrm{n}}, P_{2 \mathrm{n}}(10)$ is equal to zero

$$
\Delta=\left|\begin{array}{cc}
\alpha-\omega_{n}^{2} & -i \beta \omega_{n} \\
i \beta \omega_{n} & \alpha-\omega_{n}^{2}
\end{array}\right|=\left(\alpha-\omega_{n}^{2}\right)^{2}-\beta^{2} \omega_{n}^{2}=0 .
$$

According to Kronecker-Kapelly's theorem, the set of linear algebraic equations is compatible if and only if the matrix rank of the linear set is equal to the extended matrix rank. Therefore, the solvability conditions are

$$
\left|\begin{array}{cc}
R_{1 n} & -i \beta \omega_{n} \\
R_{2 n} & \left(\alpha-\omega_{n}^{2}\right)
\end{array}\right|=0 \quad(\mathrm{n}=1,2)
$$


otherwise the set of linear algebraic equations (10) has no solutions.

So,

$$
R_{1 n}=\frac{i \beta \omega_{n} R_{2 n}}{\omega_{n}^{2}-\alpha}
$$

and the solvability conditions can be written in the form

$$
R_{1 n}=\frac{R_{2 n}}{\bar{\Lambda}_{n}} \quad(\mathrm{n}=1,2)
$$

The differential equations to define $A_{1}\left(T_{1}\right)$ and $A_{2}\left(T_{1}\right)$ are the consequence of solvability conditions (11)

$$
\begin{aligned}
& \left(\beta \Lambda_{1}-2 i \omega_{1}+\frac{2 i \omega_{1} \Lambda_{1}+\beta}{\bar{\Lambda}_{1}}\right) A_{1}^{\prime}+\left(\frac{2 i \omega_{1} \Lambda_{1} \mu_{2}}{\bar{\Lambda}_{1}}-2 i \omega_{1} \mu_{1}\right) A_{1}=0 \\
& \left(\beta \Lambda_{2}-2 i \omega_{2}+\frac{2 i \omega_{2} \Lambda_{2}+\beta}{\bar{\Lambda}_{2}}\right) A_{2}^{\prime}+\left(\frac{2 i \omega_{2} \Lambda_{2} \mu_{2}}{\bar{\Lambda}_{2}}-2 i \omega_{2} \mu_{1}\right) A_{2}=0
\end{aligned}
$$

It follows from (3), (6) and (12) that the complex solution of the differential set (2) is

$$
\begin{aligned}
& x=\varepsilon\left[\exp \left(-\varepsilon v_{1} t\right) a_{1} \exp \left(i \omega_{1} t\right)+\exp \left(-\varepsilon v_{2} t\right) a_{2} \exp \left(i \omega_{2} t\right)+\Phi_{1} \exp [i(\Omega t+\tau)]+C C\right]+O\left(\varepsilon^{2}\right), \\
& y=\varepsilon\left[\Lambda_{1} \exp \left(-\varepsilon v_{1} t\right) a_{1} \exp \left(i \omega_{1} t\right)+\Lambda_{2} \exp \left(-\varepsilon v_{2} t\right) a_{2} \exp \left(i \omega_{2} t\right)+\Phi_{2} \exp [i(\Omega t+\tau)]+C C\right]+O\left(\varepsilon^{2}\right) .
\end{aligned}
$$

Then the real solution is as follows

$$
\begin{aligned}
& x=\varepsilon\left[\exp \left(-\varepsilon v_{1} t\right) a_{1} \cos \left(\omega_{1} t+\Theta_{1}\right)+\exp \left(-\varepsilon v_{2} t\right) a_{2} \cos \left(\omega_{2} t+\Theta_{2}\right)+2 \operatorname{Im} \Phi_{1} \sin (\Omega t+\tau)\right]+O\left(\varepsilon^{2}\right), \\
& y=\varepsilon\left[\operatorname{Im} \Lambda_{1} \exp \left(-\varepsilon v_{1} t\right) a_{1} \sin \left(\omega_{1} t+\Theta_{1}\right)+\operatorname{Im} \Lambda_{2} \exp \left(-\varepsilon v_{2} t\right) \times\right. \\
& \left.a_{2} \sin \left(\omega_{2} t+\Theta_{2}\right)+2 \Phi_{2} \cos (\Omega t+\tau)\right]+O\left(\varepsilon^{2}\right),
\end{aligned}
$$

where $v_{n}=\frac{2 \omega_{n}\left(\mu_{1}+\mu_{2}\right)}{4 \omega_{n}-\beta\left(\operatorname{Im} \Lambda_{n}+\frac{1}{\operatorname{Im} \Lambda_{n}}\right)}, a_{\mathrm{n}}$ and $\Theta_{\mathrm{n}}$ are the real constants.

Figure 2 shows a comparison of the numerical integration of (2) and the perturbation solutions (13). The following parameters of set (2) were accepted for all cases (a), (b), (c) $\alpha=1500, \quad \beta=70, \quad \alpha_{1}=9.985 \times 10^{2}, \quad \alpha_{2}=2 \times 10^{3}, \quad \alpha_{3}=7.9588 \times 10^{3}, \quad \alpha_{4}=0.002, \quad \alpha_{5}=-4.0794 \times 10^{3}$, $\alpha_{6}=4.0002 \times 10^{3}, \alpha_{7}=8.0005 \times 10^{3}, \beta_{1}=29.9975, \beta_{2}=-0.001, \beta_{3}=-4.1594 \times 10^{3}, \beta_{4}=-1.9997 \times 10^{3}, \beta_{5}=-$ $7.9188 \times 10^{3}, \beta_{6}=0.7959, \beta_{7}=-0.4083$; initial conditions are the following $x(0)=10^{-12}, y(0)=10^{-10}$, $\dot{x}(0)=\dot{y}(0)=0$.

In the case of non-resonant undamped vibrations of the rotor (Fig. 2 (a)) it is accepted for numerical integration that $\hat{\mu}_{1}=0, \hat{\mu}_{2}=0, F=0$. According to (13), the perturbation solution is presented by the expressions

$$
x=8.2686044 \cdot 10^{-6} \cos (17.2015 t)+1.6313956 \cdot 10^{-6} \cos (87.2015 t),
$$




$$
y=8.2686044 \cdot 10^{-6} \sin (17.2015 t)-1.6313956 \cdot 10^{-6} \sin (87.2015 t) \text {. }
$$

Fig. 2 (b) corresponds to the non-resonant damped vibrations of the rotor. For this case $\hat{\mu}_{1}=0.1, \hat{\mu}_{2}=0.15, F=0$. The perturbation solution has the form

$$
\begin{aligned}
& x=8.2686044 \cdot 10^{-6} \exp (-0.0412 t) \cos (17.2015 t)+1.6313956 \cdot 10^{-6} \exp (-0.2088 t) \cos (87.2015 t), \\
& y=8.2686044 \cdot 10^{-6} \exp (-0.0412 t) \sin (17.2015 t)-1.6313956 \cdot 10^{-6} \exp (-0.2088 t) \sin (87.2015 t) .
\end{aligned}
$$

For the non-resonant forced damped vibrations of the rotor (Fig. 2 (c)) it is accepted for numerical integration that $\hat{\mu}_{1}=0.1, \hat{\mu}_{2}=0.15, F=0.005, \Omega=10, \tau=-\pi / 2$. The perturbation solution is

$$
\begin{gathered}
x=5.8241 \cdot 10^{-6} \exp (-0.0412 t) \cos (17.2015 t)+1.69495 \cdot 10^{-6} \exp (-0.2088 t) \cos (87.2015 t)- \\
2.38095 \cdot 10^{-6} \sin (10 t-\pi / 2), \\
y=5.8241 \cdot 10^{-6} \exp (-0.0412 t) \sin (17.2015 t)-1.69495 \cdot 10^{-6} \exp (-0.2088 t) \sin (87.2015 t)+ \\
4.7619 \cdot 10^{-6} \cos (10 t-\pi / 2) .
\end{gathered}
$$

Fig. 2 demonstrates good agreement of the numerical and analytical solutions.

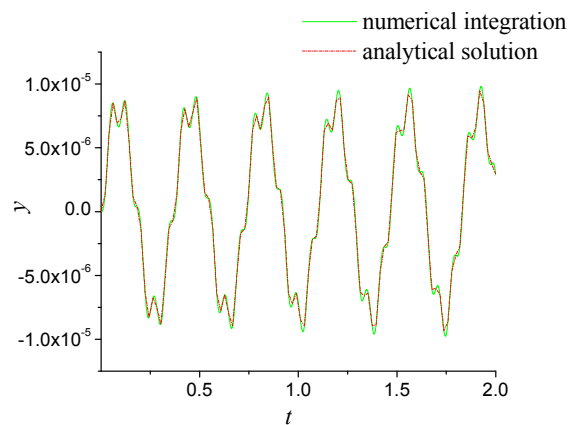

(a)

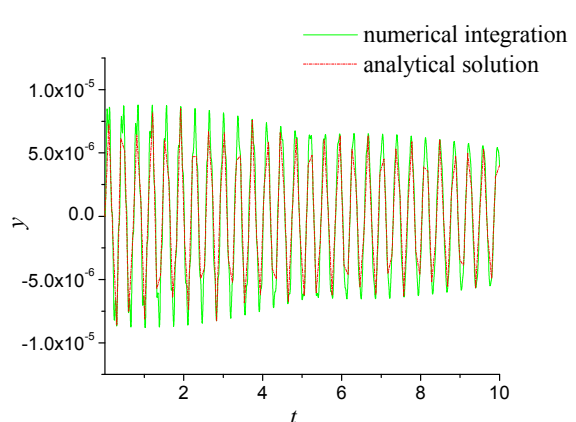

(b)

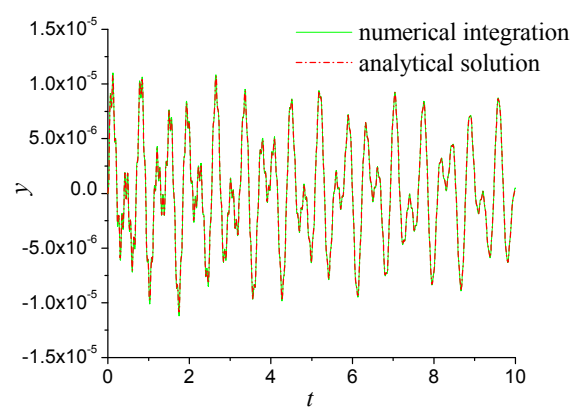

(c)

Fig. 2. Comparison of numerical integration (2) and perturbation solutions (13) in the case of (a) nonresonant undamped vibrations of the rotor, (b) nonresonant damped vibrations of the rotor; (c) nonresonant forced damped vibrations of the rotor 


\subsection{Primary resonance: The cases of no internal resonance and an internal resonance}

To analyze primary resonances the forcing term is ordered so that it appears at order $\varepsilon^{2}$ or in the same perturbation equation as the non-linear terms and damping. Thus, we recall in (2) $F=\varepsilon^{2} f, \hat{\mu}_{n}=\varepsilon \mu_{n}$. Consider the case in which $\Omega \approx \omega_{2}$. The case $\Omega \approx \omega_{1}$ is analogous. Let us introduce detuning parameter $\sigma_{1}$ and put $\Omega=\omega_{2}+\varepsilon \sigma_{1}$.

Substituting (3) into (2) and equating coefficients of similar powers of $\varepsilon$ we obtain Order $\varepsilon$

$$
\begin{aligned}
& D_{0}^{2} x_{1}+\alpha x_{1}-\beta D_{0} y_{1}=0 \\
& D_{0}^{2} y_{1}+\alpha y_{1}+\beta D_{0} x_{1}=0
\end{aligned}
$$

Order $\varepsilon^{2}$

$$
\begin{aligned}
& D_{0}^{2} x_{2}+\alpha x_{2}-\beta D_{0} y_{2}=-2 D_{0}\left(D_{1} x_{1}+\mu_{1} x_{1}\right)+\beta D_{1} y_{1}+\alpha_{1} x_{1}^{2}+\alpha_{2} y_{1}^{2}+ \\
& \alpha_{3} x_{1} D_{0} x_{1}+\alpha_{4} x_{1} y_{1}+\alpha_{5} x_{1} D_{0} y_{1}+\alpha_{6} y_{1} D_{0} x_{1}+\alpha_{7} y_{1} D_{0} y_{1} \\
& D_{0}^{2} y_{2}+\alpha y_{2}+\beta D_{0} x_{2}=-2 D_{0}\left(D_{1} y_{1}+\mu_{2} y_{1}\right)-\beta D_{1} x_{1}+\beta_{1} x_{1}^{2}+\beta_{2} y_{1}^{2}+ \\
& \beta_{3} x_{1} D_{0} x_{1}+\beta_{4} x_{1} y_{1}+\beta_{5} x_{1} D_{0} y_{1}+\beta_{6} y_{1} D_{0} x_{1}+\beta_{7} y_{1} D_{0} y_{1}+f \cos \left(\Omega T_{0}+\tau\right) .
\end{aligned}
$$

The solution of (14) is given in the form

$$
\begin{aligned}
& x_{1}=A_{1}\left(T_{1}\right) \exp \left(i \omega_{1} T_{0}\right)+A_{2}\left(T_{1}\right) \exp \left(i \omega_{2} T_{0}\right)+C C, \\
& y_{1}=\Lambda_{1} A_{1}\left(T_{1}\right) \exp \left(i \omega_{1} T_{0}\right)+\Lambda_{2} A_{2}\left(T_{1}\right) \exp \left(i \omega_{2} T_{0}\right)+C C,
\end{aligned}
$$

where $\Lambda_{n}=\frac{\omega_{n}^{2}-\alpha}{\omega_{n} \beta} i$

Substitution of (16) into (15) yields

$$
\begin{gathered}
D_{0}^{2} x_{2}+\alpha x_{2}-\beta D_{0} y_{2}=\left[-2 i \omega_{1}\left(A_{1}^{\prime}+\mu_{1} A_{1}\right)+\beta \Lambda_{1} A_{1}^{\prime}\right] \exp \left(i \omega_{1} T_{0}\right)+ \\
{\left[-2 i \omega_{2}\left(A_{2}^{\prime}+\mu_{1} A_{2}\right)+\beta \Lambda_{2} A_{2}^{\prime}\right] \exp \left(i \omega_{2} T_{0}\right)+} \\
A_{1}^{2}\left[\alpha_{1}+\Lambda_{1}^{2} \alpha_{2}+i \omega_{1} \alpha_{3}+\Lambda_{1} \alpha_{4}+i \omega_{1} \Lambda_{1} \alpha_{5}+i \omega_{1} \Lambda_{1} \alpha_{6}+i \omega_{1} \Lambda_{1}^{2} \alpha_{7}\right] \exp \left(2 i \omega_{1} T_{0}\right)+ \\
A_{2}^{2}\left[\alpha_{1}+\Lambda_{2}^{2} \alpha_{2}+i \omega_{2} \alpha_{3}+\Lambda_{2} \alpha_{4}+i \omega_{2} \Lambda_{2} \alpha_{5}+i \omega_{2} \Lambda_{2} \alpha_{6}+i \omega_{2} \Lambda_{2}^{2} \alpha_{7}\right] \exp \left(2 i \omega_{2} T_{0}\right)+ \\
A_{1} A_{2}\left[2 \alpha_{1}+2 \Lambda_{1} \Lambda_{2} \alpha_{2}+\left(i \omega_{1}+i \omega_{2}\right) \alpha_{3}+\left(\Lambda_{1}+\Lambda_{2}\right) \alpha_{4}+\left(i \omega_{2} \Lambda_{2}-i \omega_{1} \Lambda_{1}\right) \alpha_{5}+\right. \\
\left.\left(i \omega_{2} \Lambda_{1}+i \omega_{1} \Lambda_{2}\right) \alpha_{6}+\left(i \omega_{1}+i \omega_{2}\right) \Lambda_{1} \Lambda_{2} \alpha_{7}\right] \exp \left(i\left(\omega_{1}+\omega_{2}\right) T_{0}\right)+ \\
\bar{A}_{1} A_{2}\left[2 \alpha_{1}+2 \bar{\Lambda}_{1} \Lambda_{2} \alpha_{2}+\left(i \omega_{2}-i \omega_{1}\right) \alpha_{3}+\left(\Lambda_{2}+\bar{\Lambda}_{1}\right) \alpha_{4}+\left(i \omega_{2} \Lambda_{2}-i \omega_{1} \bar{\Lambda}_{1}\right) \alpha_{5}+\right. \\
\left.\left(i \omega_{2} \bar{\Lambda}_{1}-i \omega_{1} \Lambda_{2}\right) \alpha_{6}+\left(i \omega_{2}-i \omega_{1}\right) \bar{\Lambda}_{1} \Lambda_{2} \alpha_{7}\right] \exp \left(i\left(\omega_{2}-\omega_{1}\right) T_{0}\right)+ \\
A_{1} \bar{A}_{1}\left(\alpha_{1}+\Lambda_{1}\left(\bar{\Lambda}_{1} \alpha_{2}+\alpha_{4}+i \omega_{1}\left(\alpha_{5}-\alpha_{6}\right)\right)\right)+A_{2} \bar{A}_{2}\left(\alpha_{1}+\Lambda_{2}\left(\bar{\Lambda}_{2} \alpha_{2}+\alpha_{4}+i \omega_{2}\left(\alpha_{5}-\alpha_{6}\right)\right)\right)+C C,
\end{gathered}
$$




$$
\begin{aligned}
& D_{0}^{2} y_{2}+\alpha y_{2}+\beta D_{0} x_{2}=\left[-2 i \omega_{1} \Lambda_{1}\left(A_{1}^{\prime}+\mu_{2} A_{1}\right)-\beta A_{1}^{\prime}\right] \exp \left(i \omega_{1} T_{0}\right)+ \\
& \quad\left[-2 i \omega_{2} \Lambda_{2}\left(A_{2}^{\prime}+\mu_{2} A_{2}\right)-\beta A_{2}^{\prime}\right] \exp \left(i \omega_{2} T_{0}\right)+ \\
& A_{1}^{2}\left[\beta_{1}+\Lambda_{1}^{2} \beta_{2}+i \omega_{1} \beta_{3}+\Lambda_{1} \beta_{4}+i \omega_{1} \Lambda_{1} \beta_{5}+i \omega_{1} \Lambda_{1} \beta_{6}+i \omega_{1} \Lambda_{1}^{2} \beta_{7}\right] \exp \left(2 i \omega_{1} T_{0}\right)+ \\
& \quad A_{2}^{2}\left[\beta_{1}+\Lambda_{2}^{2} \beta_{2}+i \omega_{2} \beta_{3}+\Lambda_{2} \beta_{4}+i \omega_{2} \Lambda_{2} \beta_{5}+i \omega_{2} \Lambda_{2} \beta_{6}+i \omega_{2} \Lambda_{2}^{2} \beta_{7}\right] \exp \left(2 i \omega_{2} T_{0}\right)+ \\
& A_{1} A_{2}\left[2 \beta_{1}+2 \Lambda_{1} \Lambda_{2} \beta_{2}+\left(i \omega_{1}+i \omega_{2}\right) \beta_{3}+\left(\Lambda_{1}+\Lambda_{2}\right) \beta_{4}+\left(i \omega_{2} \Lambda_{2}-i \omega_{1} \Lambda_{1}\right) \beta_{5}+\right. \\
& \left.\left(i \omega_{2} \Lambda_{1}+i \omega_{1} \Lambda_{2}\right) \beta_{6}+\left(i \omega_{1}+i \omega_{2}\right) \Lambda_{1} \Lambda_{2} \beta_{7}\right] \exp \left(i\left(\omega_{1}+\omega_{2}\right) T_{0}\right)+ \\
& \bar{A}_{1} A_{2}\left[2 \beta_{1}+2 \bar{\Lambda}_{1} \Lambda_{2} \beta_{2}+\left(i \omega_{2}-i \omega_{1}\right) \beta_{3}+\left(\Lambda_{2}+\bar{\Lambda}_{1}\right) \beta_{4}+\left(i \omega_{2} \Lambda_{2}-i \omega_{1} \bar{\Lambda}_{1}\right) \beta_{5}+\right. \\
& \left.\left(i \omega_{2} \bar{\Lambda}_{1}-i \omega_{1} \Lambda_{2}\right) \beta_{6}+\left(i \omega_{2}-i \omega_{1}\right) \bar{\Lambda}_{1} \Lambda_{2} \beta_{7}\right] \exp \left(i\left(\omega_{2}-\omega_{1}\right) T_{0}\right)+ \\
& A_{1} \bar{A}_{1}\left(\beta_{1}+\Lambda_{1}\left(\bar{\Lambda}_{1} \beta_{2}+\beta_{4}+i \omega_{1}\left(\beta_{5}-\beta_{6}\right)\right)\right)+A_{2} \bar{A}_{2}\left(\beta_{1}+\Lambda_{2}\left(\bar{\Lambda}_{2} \beta_{2}+\beta_{4}+i \omega_{2}\left(\beta_{5}-\beta_{6}\right)\right)\right)+ \\
& \frac{1}{2} f \exp \left(i\left(\omega_{2} T_{0}+\sigma_{1} T_{1}+\tau\right)\right)+C C .
\end{aligned}
$$

Let $\omega_{2}>\omega_{1}$ for definiteness. We need to distinguish between the case of internal resonance $\omega_{2} \approx 2 \omega_{1}$ and the case of no internal resonance, i.e., $\omega_{2}$ is away from $2 \omega_{1}$. The case $\omega_{1}>\omega_{2}$, $\omega_{1} \approx 2 \omega_{2}$ is analogous. When $\omega_{2}$ is away from $2 \omega_{1}$ the solvability conditions (11) are written in the form

$$
\begin{aligned}
& q_{\omega_{1}}+\frac{1}{\Lambda_{1}} p_{\omega_{1}}=0, \\
& q_{\omega_{2}}+\frac{1}{\Lambda_{2}} p_{\omega_{2}}+\frac{1}{2 \Lambda_{2}} f \exp \left(i\left(\sigma_{1} T_{1}+\tau\right)\right)=0,
\end{aligned}
$$

where

$$
\begin{gathered}
q_{\omega_{1}}=-2 i \omega_{1}\left(A_{1}^{\prime}+\mu_{1} A_{1}\right)+\beta \Lambda_{1} A_{1}^{\prime}, \quad q_{\omega_{2}}=2 i \omega_{2}\left(A_{2}^{\prime}+\mu_{1} A_{2}\right)+\beta \Lambda_{2} A_{2}^{\prime}, \\
p_{\omega_{1}}=-2 i \omega_{1} \Lambda_{1}\left(A_{1}^{\prime}+\mu_{2} A_{1}\right)-\beta A_{1}^{\prime}, \quad p_{\omega_{2}}=-2 i \omega_{2} \Lambda_{2}\left(A_{2}^{\prime}+\mu_{2} A_{2}\right)-\beta A_{2}^{\prime} .
\end{gathered}
$$

Thus, when there is no internal resonance, the first approximation is not influenced by the non-linear terms; it is essentially a solution of the corresponding linear problem.

Actually, the solutions of the differential equations below

$$
\begin{aligned}
& \left(\beta \Lambda_{1}-2 i \omega_{1}+\frac{2 i \omega_{1} \Lambda_{1}+\beta}{\bar{\Lambda}_{1}}\right) A_{1}^{\prime}+\left(\frac{2 i \omega_{1} \Lambda_{1} \mu_{2}}{\bar{\Lambda}_{1}}-2 i \omega_{1} \mu_{1}\right) A_{1}=0 \\
& \left(\beta \Lambda_{2}-2 i \omega_{2}+\frac{2 i \omega_{2} \Lambda_{2}+\beta}{\bar{\Lambda}_{2}}\right) A_{2}^{\prime}+\left(\frac{2 i \omega_{2} \Lambda_{2} \mu_{2}}{\bar{\Lambda}_{2}}-2 i \omega_{2} \mu_{1}\right) A_{2}=-\frac{1}{2 \Lambda_{2}} f \exp \left[i\left(\sigma_{1} T_{1}+\tau\right)\right]
\end{aligned}
$$

are

$$
A_{1}\left(T_{1}\right)=\frac{1}{2} a_{1} \exp \left(-v_{1} T_{1}+i \Theta_{1}\right)
$$




$$
A_{2}\left(T_{1}\right)=\frac{1}{2} a_{2} \exp \left(-v_{2} T_{1}+i \Theta_{2}\right)+\frac{f\left(v_{2}-i \sigma_{1}\right)}{2 \operatorname{Im} \Lambda_{2} \operatorname{Im} \kappa_{2}\left(v_{2}^{2}+\sigma_{1}^{2}\right)} \exp \left[i\left(\sigma_{1} T_{1}+\tau\right)\right],
$$

where $a_{\mathrm{n}}$ and $\Theta_{\mathrm{n}}$ are the real constants,

$$
v_{n}=\frac{2 \omega_{n}\left(\mu_{1}+\mu_{2}\right)}{4 \omega_{n}-\beta\left(\operatorname{Im} \Lambda_{n}+\frac{1}{\operatorname{Im} \Lambda_{n}}\right)}, \kappa_{2}=-4 \omega_{2} i+\beta\left(\operatorname{Im} \Lambda_{2}+\frac{1}{\operatorname{Im} \Lambda_{2}}\right) i
$$

As $\mathrm{t} \rightarrow \infty, \mathrm{T}_{1} \rightarrow \infty$ and

$$
A_{1} \rightarrow 0, \quad A_{2} \rightarrow \frac{f\left(v_{2}-i \sigma_{1}\right)}{2 \operatorname{Im} \Lambda_{2} \operatorname{Im} \kappa_{2}\left(v_{2}^{2}+\sigma_{1}^{2}\right)} \exp \left[i\left(\sigma_{1} T_{1}+\tau\right)\right]
$$

according to (16), we obtain the following steady-state response:

$$
\begin{gathered}
x_{1}=\frac{f\left(v_{2}-i \sigma_{1}\right)}{2 \operatorname{Im} \Lambda_{2} \operatorname{Im} \kappa_{2}\left(v_{2}^{2}+\sigma_{1}^{2}\right)} \exp \left[i\left(\omega_{2} T_{0}+\sigma_{1} T_{1}+\tau\right)\right]+C C, \\
y_{1}=\Lambda_{2} \frac{f\left(v_{2}-i \sigma_{1}\right)}{2 \operatorname{Im} \Lambda_{2} \operatorname{Im} \kappa_{2}\left(v_{2}^{2}+\sigma_{1}^{2}\right)} \exp \left[i\left(\omega_{2} T_{0}+\sigma_{1} T_{1}+\tau\right)\right]+C C .
\end{gathered}
$$

Therefore, the real solution is

$$
\begin{aligned}
& x=\frac{F}{\varepsilon} \frac{1}{\operatorname{Im} \Lambda_{2} \operatorname{Im} \kappa_{2}\left(v_{2}^{2}+\sigma_{1}^{2}\right)}\left[v_{2} \cos (\Omega t+\tau)+\sigma_{1} \sin (\Omega t+\tau)\right]+O\left(\varepsilon^{2}\right), \\
& y=\frac{F}{\varepsilon} \frac{1}{\operatorname{Im} \kappa_{2}\left(v_{2}^{2}+\sigma_{1}^{2}\right)}\left[\sigma_{1} \cos (\Omega t+\tau)-v_{2} \sin (\Omega t+\tau)\right]+O\left(\varepsilon^{2}\right),
\end{aligned}
$$

or it can be rewritten in the form

$$
\begin{aligned}
& x=\frac{F}{\varepsilon} \frac{1}{\operatorname{Im} \Lambda_{2} \operatorname{Im} \kappa_{2}\left(v_{2}^{2}+\sigma_{1}^{2}\right)^{1 / 2}} \sin \left(\Omega t+\tau+\tilde{\gamma}_{1}\right)+O\left(\varepsilon^{2}\right), \\
& y=\frac{F}{\varepsilon} \frac{1}{\operatorname{Im} \kappa_{2}\left(v_{2}^{2}+\sigma_{1}^{2}\right)^{1 / 2}} \sin \left(\Omega t+\tau+\tilde{\gamma}_{2}\right)+O\left(\varepsilon^{2}\right),
\end{aligned}
$$

where $\tilde{\gamma}_{1}=\operatorname{arctg}\left(v_{2} / \sigma_{1}\right), \tilde{\gamma}_{2}=-\operatorname{arctg}\left(\sigma_{1} / v_{2}\right)$.

Other situation occurs when the internal resonance $\omega_{2} \approx 2 \omega_{1}$ exists. Let us introduce detuning parameter $\sigma_{2}$ and put $\omega_{2}=2 \omega_{1}-\varepsilon \sigma_{2}$.

Taking into account (11), the solvability conditions for this case become

$$
\begin{aligned}
& q_{\omega_{1}}+\frac{1}{\Lambda_{1}} p_{\omega_{1}}+\left(q_{\omega_{2}-\omega_{1}}+\frac{1}{\Lambda_{1}} p_{\omega_{2}-\omega_{1}}\right) \bar{A}_{1} A_{2} \exp \left(-i \sigma_{2} T_{1}\right)=0, \\
& q_{\omega_{2}}+\frac{1}{\Lambda_{2}} p_{\omega_{2}}+\left(q_{2 \omega_{1}}+\frac{1}{\Lambda_{2}} p_{2 \omega_{1}}\right) A_{1}^{2} \exp \left(i \sigma_{2} T_{1}\right)+\frac{1}{2 \Lambda_{2}} f \exp \left(i\left(\sigma_{1} T_{1}+\tau\right)\right)=0 .
\end{aligned}
$$


Here coefficients $q_{\omega 1}, q_{\omega 2}, q_{\omega 2-\omega 1}, q_{2 \omega 1}$ are the expressions in the bracket at the exponents with the corresponding powers (17) and $p_{\omega 1}, p_{\omega 2}, p_{\omega 2-\omega 1}, p_{2 \omega 1}$ are the expressions in the bracket at the exponents with the corresponding powers (18):

$$
\begin{gathered}
q_{\omega_{1}}=-2 i \omega_{1}\left(A_{1}^{\prime}+\mu_{1} A_{1}\right)+\beta \Lambda_{1} A_{1}^{\prime}, \quad q_{\omega_{2}}=2 i \omega_{2}\left(A_{2}^{\prime}+\mu_{1} A_{2}\right)+\beta \Lambda_{2} A_{2}^{\prime}, \\
q_{2 \omega_{1}}=\alpha_{1}+\Lambda_{1}^{2} \alpha_{2}+i \omega_{1} \alpha_{3}+\Lambda_{1} \alpha_{4}+i \omega_{1} \Lambda_{1} \alpha_{5}+i \omega_{1} \Lambda_{1} \alpha_{6}+i \omega_{1} \Lambda_{1}^{2} \alpha_{7} \\
q_{\omega_{2}-\omega_{1}}=2 \alpha_{1}+2 \bar{\Lambda}_{1} \Lambda_{2} \alpha_{2}+\left(i \omega_{2}-i \omega_{1}\right) \alpha_{3}+\left(\Lambda_{2}+\bar{\Lambda}_{1}\right) \alpha_{4}+\left(i \omega_{2} \Lambda_{2}-i \omega_{1} \bar{\Lambda}_{1}\right) \alpha_{5}+ \\
\left(i \omega_{2} \bar{\Lambda}_{1}-i \omega_{1} \Lambda_{2}\right) \alpha_{6}+\left(i \omega_{2}-i \omega_{1}\right) \bar{\Lambda}_{1} \Lambda_{2} \alpha_{7}, \\
p_{\omega_{1}}=-2 i \omega_{1} \Lambda_{1}\left(A_{1}^{\prime}+\mu_{2} A_{1}\right)-\beta A_{1}^{\prime}, \quad p_{\omega_{2}}=-2 i \omega_{2} \Lambda_{2}\left(A_{2}^{\prime}+\mu_{2} A_{2}\right)-\beta A_{2}^{\prime}, \\
p_{2 \omega_{1}}=\beta_{1}+\Lambda_{1}^{2} \beta_{2}+i \omega_{1} \beta_{3}+\Lambda_{1} \beta_{4}+i \omega_{1} \Lambda_{1} \beta_{5}+i \omega_{1} \Lambda_{1} \beta_{6}+i \omega_{1} \Lambda_{1}^{2} \beta_{7}, \\
p_{\omega_{2}-\omega_{1}}=2 \beta_{1}+2 \bar{\Lambda}_{1} \Lambda_{2} \beta_{2}+\left(i \omega_{2}-i \omega_{1}\right) \beta_{3}+\left(\Lambda_{2}+\bar{\Lambda}_{1}\right) \beta_{4}+\left(i \omega_{2} \Lambda_{2}-i \omega_{1} \bar{\Lambda}_{1}\right) \beta_{5}+ \\
\left(i \omega_{2} \bar{\Lambda}_{1}-i \omega_{1} \Lambda_{2}\right) \beta_{6}+\left(i \omega_{2}-i \omega_{1}\right) \bar{\Lambda}_{1} \Lambda_{2} \beta_{7} .
\end{gathered}
$$

For the convenience let us introduce the polar notation

$$
A_{m}=\frac{1}{2} a_{m} \exp \left(i \Theta_{m}\right), \quad m=1,2 ，
$$

where $a_{\mathrm{m}}$ and $\Theta_{\mathrm{m}}$ are the real functions of $T_{1}$.

Substitution of (22) into (21) yields

$$
\begin{aligned}
& \left(a_{1}^{\prime}+i a_{1} \Theta_{1}^{\prime}\right)+v_{1} a_{1}+\frac{1}{2 \kappa_{1}} a_{1} a_{2}[\varphi+i \psi] \exp \left(i \gamma_{2}\right)=0, \\
& \left(a_{2}^{\prime}+i a_{2} \Theta_{2}^{\prime}\right)+v_{2} a_{2}+\frac{1}{2 \kappa_{2}} a_{1}^{2}[\zeta+i \eta] \exp \left(-i \gamma_{2}\right)+\frac{f}{\kappa_{2} \Lambda_{2}} \exp \left(i \gamma_{1}\right)=0 .
\end{aligned}
$$

In the expressions above the following notations were introduced

$$
\begin{gathered}
\varphi=\operatorname{Re}\left(q_{\omega_{2}-\omega_{1}}+\frac{1}{\Lambda_{1}} p_{\omega_{2}-\omega_{1}}\right), \psi=\operatorname{Im}\left(q_{\omega_{2}-\omega_{1}}+\frac{1}{\Lambda_{1}} p_{\omega_{2}-\omega_{1}}\right), \zeta=\operatorname{Re}\left(q_{2 \omega_{1}}+\frac{1}{\Lambda_{2}} p_{2 \omega_{1}}\right) \\
\eta=\operatorname{Im}\left(q_{2 \omega_{1}}+\frac{1}{\Lambda_{2}} p_{2 \omega_{1}}\right), \kappa_{n}=-4 \omega_{n} i+\beta\left(\operatorname{Im} \Lambda_{n}+\frac{1}{\operatorname{Im} \Lambda_{n}}\right) i, \quad n=1,2 \\
\gamma_{1}=\sigma_{1} T_{1}+\tau-\Theta_{2}, \quad \gamma_{2}=\Theta_{2}-2 \Theta_{1}-\sigma_{2} T_{1}
\end{gathered}
$$

$v_{1}$ and $v_{2}$ are defined as in Eq (13).

Separating Eqs. (23) into real and imaginary parts and taking into account that according to (6) $\Lambda_{\mathrm{n}}(n=1,2)$ is the imaginary value, we obtain 


$$
\begin{gathered}
a_{1}^{\prime}=-v_{1} a_{1}-\frac{a_{1} a_{2}}{2 \operatorname{Im} \kappa_{1}}\left(\psi \cos \gamma_{2}+\varphi \sin \gamma_{2}\right), \\
a_{1} \Theta_{1}^{\prime}=\frac{a_{1} a_{2}}{2 \operatorname{Im} \kappa_{1}}\left(\varphi \cos \gamma_{2}-\psi \sin \gamma_{2}\right), \\
a_{2}^{\prime}=-v_{2} a_{2}-\frac{a_{1}^{2}}{2 \operatorname{Im} \kappa_{2}}\left(\eta \cos \gamma_{2}-\zeta \sin \gamma_{2}\right)+\frac{f}{\operatorname{Im} \kappa_{2} \operatorname{Im} \Lambda_{2}} \cos \gamma_{1}, \\
a_{2} \Theta_{2}{ }^{\prime}=\frac{a_{1}^{2}}{2 \operatorname{Im} \kappa_{2}}\left(\zeta \cos \gamma_{2}+\eta \sin \gamma_{2}\right)+\frac{f}{\operatorname{Im} \kappa_{2} \operatorname{Im} \Lambda_{2}} \sin \gamma_{1} .
\end{gathered}
$$

For the steady-state response $a_{n}^{\prime}=\gamma_{n}^{\prime}=0$, therefore $\Theta_{1}{ }^{\prime}=\frac{1}{2}\left(\sigma_{1}-\sigma_{2}\right), \Theta_{2}{ }^{\prime}=\sigma_{1}$.

Two possibilities follow from (24). The first one is given by (19). It is the solution of the linear problem. Let us find functions $a_{1}$ and $a_{2}$ of $T_{1}$ according to the second possibility. It follows from the first two Eqs. (24) that

$$
\begin{gathered}
\frac{4 \omega_{1}\left(\mu_{1}+\mu_{2}\right)}{a_{2}}=-\psi \cos \gamma_{2}-\varphi \sin \gamma_{2}, \\
\frac{\operatorname{Im} \kappa_{1}}{a_{2}}\left(\sigma_{1}-\sigma_{2}\right)=\varphi \cos \gamma_{2}-\psi \sin \gamma_{2} .
\end{gathered}
$$

So,

$$
a_{2}=\left(\frac{16 \omega_{1}^{2}\left(\mu_{1}+\mu_{2}\right)^{2}+\operatorname{Im} \kappa_{1}^{2}\left(\left(\sigma_{1}-\sigma_{2}\right)^{2}\right)}{\varphi^{2}+\psi^{2}}\right)^{1 / 2}
$$

Let us take $\sin \gamma_{2}$ and $\cos \gamma_{2}$ using, for example, the formulas by Cramer

$$
\begin{gathered}
\cos \gamma_{2}=\frac{\Delta_{1}}{\Delta}, \quad \sin \gamma_{2}=\frac{\Delta_{2}}{\Delta}, \text { where } \Delta=\left|\begin{array}{cc}
-\psi & -\varphi \\
\varphi & -\psi
\end{array}\right|=\varphi^{2}+\psi^{2}, \\
\Delta_{1}=-\frac{1}{a_{2}}\left|\begin{array}{cc}
2 \operatorname{Im} \kappa_{1} v_{1} & \varphi \\
\operatorname{Im} \kappa_{1}\left(\sigma_{1}-\sigma_{2}\right) & \psi
\end{array}\right|=\frac{1}{a_{2}}\left(4 \omega_{1}\left(\mu_{1}+\mu_{2}\right) \psi+\operatorname{Im} \kappa_{1}\left(\sigma_{1}-\sigma_{2}\right) \varphi\right), \\
\Delta_{2}=\frac{1}{a_{2}}\left|\begin{array}{cc}
-\psi & 2 \operatorname{Im} \kappa_{1} v_{1} \\
\varphi & \operatorname{Im} \kappa_{1}\left(\sigma_{1}-\sigma_{2}\right)
\end{array}\right|=\frac{1}{a_{2}}\left(-\operatorname{Im} \kappa_{1}\left(\sigma_{1}-\sigma_{2}\right) \psi-4 \omega_{1}\left(\mu_{1}+\mu_{2}\right) \varphi\right) .
\end{gathered}
$$

Then a biquadratic equation relative to $a_{1}$ follows from the last two Eqs. (24)

$$
a_{1}^{4}\left(\zeta^{2}+\eta^{2}\right)+4 a_{1}^{2}\left[-2 \omega_{2}\left(\mu_{1}+\mu_{2}\right) a_{2}\left(\eta \cos \gamma_{2}-\zeta \sin \gamma_{2}\right)-\operatorname{Im} \kappa_{2} \sigma_{1} a_{2}\left(\zeta \cos \gamma_{2}+\eta \sin \gamma_{2}\right)\right]+
$$




$$
4\left[4 \omega_{2}^{2}\left(\mu_{1}+\mu_{2}\right)^{2}+\operatorname{Im} \kappa_{2}^{2} \sigma_{1}^{2}\right] a_{2}^{2}-\frac{4 f^{2}}{\left(\operatorname{Im} \Lambda_{2}\right)^{2}}=0 .
$$

Finally, we obtain the expression for $a_{1}$ :

$$
a_{1}=\left[-\frac{p}{2} \pm\left(\left(\frac{p}{2}\right)^{2}-q\right)^{\frac{1}{2}}\right]^{\frac{1}{2}}
$$

where

$$
\begin{gathered}
p=\frac{4 a_{2}}{\zeta^{2}+\eta^{2}}\left[-2 \omega_{2}\left(\mu_{1}+\mu_{2}\right)\left(\eta \cos \gamma_{2}-\zeta \sin \gamma_{2}\right)-\operatorname{Im} \kappa_{2} \sigma_{1}\left(\zeta \cos \gamma_{2}+\eta \sin \gamma_{2}\right)\right], \\
q=\frac{1}{\zeta^{2}+\eta^{2}}\left\{4 a_{2}^{2}\left[4 \omega_{2}^{2}\left(\mu_{1}+\mu_{2}\right)^{2}+\operatorname{Im} \kappa_{2}^{2} \sigma_{1}^{2}\right]-\frac{4 f^{2}}{\left(\operatorname{Im} \Lambda_{2}\right)^{2}}\right\} .
\end{gathered}
$$

Thus, the unknown functions in (16) were defined. It follows from (3), (16) and (22) that

$$
\begin{aligned}
& x=\varepsilon\left[\frac{1}{2} a_{1} \exp \left[i\left(\Theta_{1}+\omega_{1} T_{0}\right)\right]+\frac{1}{2} a_{2} \exp \left[i\left(\Theta_{2}+\omega_{2} T_{0}\right)\right]+C C\right]+O\left(\varepsilon^{2}\right), \\
& y=\varepsilon\left[\frac{1}{2} \Lambda_{1} a_{1} \exp \left[i\left(\Theta_{1}+\omega_{1} T_{0}\right)\right]+\frac{1}{2} \Lambda_{2} a_{2} \exp \left[i\left(\Theta_{2}+\omega_{2} T_{0}\right)\right]+C C\right]+O\left(\varepsilon^{2}\right) .
\end{aligned}
$$

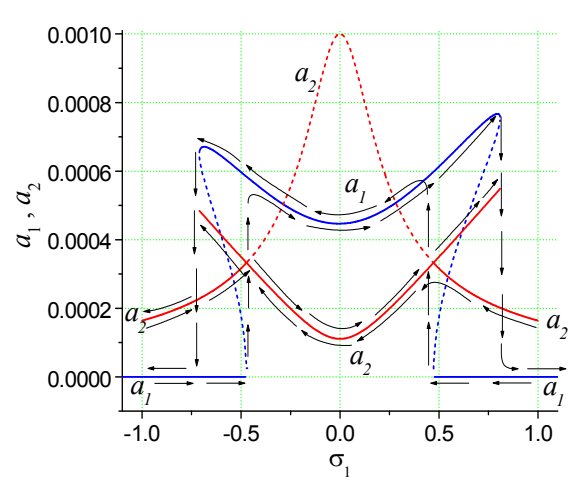

(a)

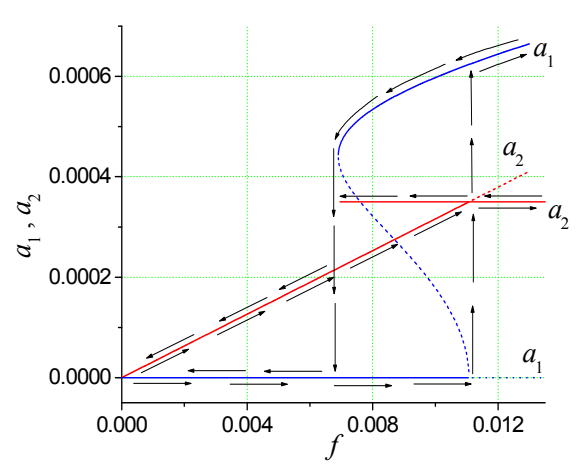

(b)

Fig. 3. (a) Frequency-response curves; $\sigma_{2}=0, \Omega \approx \omega_{2}$; (b) amplitudes $a_{1}, a_{2}$ versus the amplitude of external excitation f; $\Omega \approx \omega_{2}, \sigma_{1}=-0.5, \sigma_{2}=0$

Then, the real solution is as follows 


$$
\begin{aligned}
& x=\varepsilon\left\{a_{1} \cos \left[\frac{1}{2}\left(\Omega t+\tau-\gamma_{1}-\gamma_{2}\right)\right]+a_{2} \cos \left(\Omega t+\tau-\gamma_{1}\right)\right\}+O\left(\varepsilon^{2}\right), \\
& y=-\varepsilon\left\{a_{1} \operatorname{Im} \Lambda_{1} \sin \left[\frac{1}{2}\left(\Omega t+\tau-\gamma_{1}-\gamma_{2}\right)\right]+a_{2} \operatorname{Im} \Lambda_{2} \sin \left(\Omega t+\tau-\gamma_{1}\right)\right\}+O\left(\varepsilon^{2}\right) .
\end{aligned}
$$

Here $a_{1}$ and $a_{2}$ are defined by (25), (26).

Let us consider the expression for $a_{1}(26)$. When $\{[(p / 2)>0] \wedge(q>0)\} \vee\left[(p / 2)^{2}<q\right]$, there are no real values of $a_{1}$ defined by (26) and the response must be given by (20). When $\left[(p / 2)^{2}>q\right] \wedge(q<0)$, there is one real solution defined by (26). Therefore, the response is one of the two possibilities given by (20) and (27). When $[(p / 2)<0] \wedge\left[(p / 2)^{2}>q\right] \wedge(q>0)$, there are two real solutions defined by (26). Therefore, the response is one of the three possibilities given by (20) and (27).

In Fig. 3 (a) the frequency-response curves are depicted. $a_{1}$ and $a_{2}$ are plotted as a function of $\sigma_{1}$ for $\sigma_{2}=0$. The dashed line having a peak at $\sigma_{1}=0$ corresponds to $a_{1}=0$ and it is a solution of the corresponding linear problem. Arrows indicate the jump phenomenon associated with varying the frequency of external excitation $\Omega$. Perturbation solution obtained is the superposition of two submotions with amplitudes $a_{1}$ and $a_{2}$ and frequencies $\omega_{1}, \omega_{2}$ correspondingly. To compare the perturbation and numerical solutions we performed an approximate harmonic analysis of solutions $x(\mathrm{t}), y(\mathrm{t})$ obtained numerically. These functions are expanded in Fourier series formed of cosines

$$
x(t)=\frac{a_{0}}{2}+\sum_{k=1}^{\infty} a_{k} \cos \frac{k \pi t}{T}, a_{k}=\frac{2}{T} \int_{0}^{T} x(t) \cos \frac{k \pi t}{T} d t, k=0,1,2 \ldots,
$$

where $T$ is the period of integration, $0 \leq t \leq T$. The coefficients of the Fourier series were calculated approximately. The following parameters of set (2) were accepted: $\alpha=200, \beta=10$ (parameters $\alpha=200, \beta=10$ correspond to natural frequencies $\omega_{1}=10, \omega_{2}=20$, i.e. $\omega_{2}=2 \omega_{1}$ ), $\alpha_{1}=9.985 \times 10^{2}, \quad \alpha_{2}=2 \times 10^{3}, \quad \alpha_{3}=7.9588 \times 10^{3}, \quad \alpha_{4}=0.002, \quad \alpha_{5}=-4.0794 \times 10^{3}, \quad \alpha_{6}=4.0002 \times 10^{3}$, $\alpha_{7}=8.0005 \times 10^{3}, \quad \beta_{1}=29.9975, \quad \beta_{2}=-0.001, \quad \beta_{3}=-4.1594 \times 10^{3}, \quad \beta_{4}=-1.9997 \times 10^{3}, \quad \beta_{5}=-7.9188 \times 10^{3}$, $\beta_{6}=0.7959, \beta_{7}=-0.4083$. The perturbation and numerical solutions of (2) are in good agreement. In Fig. 3 (b) one can see saturation phenomenon. As $f$ increases from zero, $a_{2}$ increases too until it reaches the value $a_{2}=3.5 \times 10^{-4}$ while $a_{1}$ is zero. This agrees with the solution of the corresponding linear problem. Then $a_{2}$ saves the constant value and $a_{1}$ starts to increase. Approximate harmonic analysis demonstrates good agreement of the theoretical prediction presented in Fig. 3 (b) and the corresponding numerical solution of (2).

\section{Rigid magnetic materials. Conditions for chaotic vibrations of the rotor in various control parameter planes}

In the case of rigid magnetic materials the hysteretic properties of system (1) can be considered using the Bouc-Wen hysteretic model. It was shown (Awrejcewicz \& Dzyubak, 2007) that this modeling mechanism for energy dissipation was sufficiently accurate to model loops of various shapes in accordance with a real experiment, reflecting the behavior of hysteretic systems from very different fields. The hysteretic model of the rotor-MHDB system is as follows 


$$
\begin{gathered}
\ddot{x}=P_{r}(\rho, \dot{\rho}, \dot{\varphi}) \cos \varphi-P_{\tau}(\rho, \dot{\varphi}) \sin \varphi-\gamma_{m} \dot{x}-\lambda_{m}\left[\delta\left(x-x_{0}\right)+(1-\delta) z_{1}\right], \\
\ddot{y}=P_{r}(\rho, \dot{\rho}, \dot{\varphi}) \sin \varphi+P_{\tau}(\rho, \dot{\varphi}) \cos \varphi-\gamma_{m} \dot{y}-\lambda_{m}\left[\delta\left(y-y_{0}\right)+(1-\delta) z_{2}\right]+Q_{0}+Q \sin \Omega t, \\
\dot{z}_{1}=\left[k_{z}-\left(\gamma+\beta \operatorname{sgn}(\dot{x}) \operatorname{sgn}\left(z_{1}\right)\right)\left|z_{1}\right|^{n}\right] \dot{x}, \\
\dot{z}_{2}=\left[k_{z}-\left(\gamma+\beta \operatorname{sgn}(\dot{y}) \operatorname{sgn}\left(z_{2}\right)\right)\left|z_{2}\right|^{n}\right] \dot{y} .
\end{gathered}
$$

Here $z_{1}$ and $z_{2}$ are the hysteretic forces. The case $\delta=0$ corresponds to maximal hysteretic dissipation and $\delta=1$ corresponds to the absence of hysteretic forces in the system, parameters $\left(k_{z}, \beta, n\right) \in \mathrm{R}^{+}$and $\gamma \in \mathrm{R}$ govern the shape of the hysteresis loops.

Conditions for chaotic vibrations of the rotor have been found using the approach based on the analysis of the wandering trajectories. The description of the approach, its advantages over standard procedures and a comparison with other approaches can be found, for example, in (Awrejcewicz \& Dzyubak, 2007; Awrejcewicz \& Mosdorf, 2003; Awrejcewicz et al., 2005).

The stability of motion depends on all the parameters of system (28), including initial conditions. We traced the irregular vibrations of the rotor to sufficient accuracy in the parametric planes of amplitude of external excitation versus hysteretic dissipation $(\delta, Q)$, the amplitude versus frequency of external excitation $(\Omega, Q)$, the amplitude versus dynamic oilfilm action characteristics $(C, Q)$ and the amplitude versus the magnetic control parameters $\left(\gamma_{m}, Q\right),\left(\lambda_{m}, Q\right)$.

It should be noted, that chaos is not found in absence of hysteresis when $\delta=1$. Chaotic vibrations of the rotor are caused by hysteresis and for all chaotic regions presented $\delta \neq 1$. So, in system (28) chaos was quantified using the following conditions

$$
\begin{array}{cc}
\exists t^{*} \in\left[t_{1}, T\right]:\left\{\left(\left|x\left(t^{*}\right)-\tilde{x}\left(t^{*}\right)\right|>\alpha A_{x}\right) \vee\left(\left|y\left(t^{*}\right)-\tilde{y}\left(t^{*}\right)\right|>\alpha A_{y}\right)\right\} \\
\Downarrow & \Downarrow \\
\begin{array}{cc}
\Downarrow \\
\text { in the hootic vibrations }
\end{array} & \text { chaotic vibrations } \\
\text { in the vertical direction }
\end{array}
$$

Here $x(t), \tilde{x}(t)$ and $y(t), \tilde{y}(t)$ are nearby trajectories respectively, $A_{x}$ and $A_{y}$ are the characteristic vibration amplitudes of the rotor in the horizontal and vertical direction respectively

$$
A_{x}=\frac{1}{2}\left|\max _{t_{1} \leq t \leq T} x(t)-\min _{t_{1} \leq t \leq T} x(t)\right|, A_{y}=\frac{1}{2}\left|\max _{t_{1} \leq t \leq T} y(t)-\min _{t_{1} \leq t \leq T} y(t)\right| .
$$

$\left[t_{1}, T\right] \subset\left[t_{0}, T\right]$ and $\left[t_{0}, T\right]$ is the time interval over which the trajectories are considered. The interval $\left[t_{0}, t_{1}\right]$ is the time interval over which all transient processes are damped. The parameter $\alpha$ introduced is an auxiliary parameter such that $0<\alpha<1 . \alpha A_{x}, \alpha A_{y}$ are referred to as the divergence measures of the observable trajectories in the horizontal and vertical 
directions and, with the aid of the chosen parameter $\alpha$, are inadmissible for the case of regularity of the motion.

If the inequality (29) is satisfied in some nodal point of the sampled control parameter space, then the motion is chaotic (including transient and alternating chaos). The manifold of all such nodal points of the investigated control parameter space defines the domains of chaotic behaviour for the considered system.

Figure 4 (a) displays the regions of rotor chaotic vibrations in $(\delta, Q)$ plane. The part of this plane $\left(10^{-7}<\delta \leq 0.0017 ; 0.00125<Q \leq 0.00185\right)$ was sampled by means of an uniform rectangular grid. For this aim two families of straight lines were drawn through dividing points of the axes

$$
\begin{gathered}
\delta=i \Delta \delta \quad(i=0,1, \ldots, 120), \\
Q^{j}=j \Delta Q \quad(j=0,1, \ldots, 120) .
\end{gathered}
$$

Here $\Delta \delta=1.4165 \times 10^{-5}, \Delta Q=5 \times 10^{-6}$.

The time period for the simulation $\mathrm{T}$ is of $\frac{200 \pi}{\Omega}$ in nondimentional time units. During the computations, two thirds of the time period $\mathrm{T}$ corresponds to the time interval $\left[t_{0}, t_{1}\right]$, where transient processes are damped. The integration step size is $0.02 \frac{\pi}{\Omega}$. Initial conditions of the nearby trajectories are differed less than $0.5 \%$ of characteristic vibration amplitudes, e.g. the starting points of these trajectories are in the rectangle $\left(\left|x\left(t_{0}\right)-\tilde{x}\left(t_{0}\right)\right|<0.005 A_{x}\right.$, $\left.\left|y\left(t_{0}\right)-\tilde{y}\left(t_{0}\right)\right|<0.005 A_{y}\right)$. The parameter $\alpha$ is chosen to be equal to $\frac{1}{3}$.

All domains have complex structure. There are a number of scattered points, streaks and islets here. Such a structure is characteristic of domains where chaotic vibrations are possible. For each aggregate of control parameters there is some critical value of the hysteretic dissipation $\left(1-\delta_{c r}\right)$ that if $(1-\delta)<\left(1-\delta_{c r}\right)$, chaos is not observed in the system considered.

In Fig. 4 (b) chaotic regions for the vertical vibrations of the rotor are depicted in the $(\Omega, Q)$ parametric plane $(0.25<\Omega \leq 1.2 ; 0.0015<Q \leq 0.0022)$. The time period for the simulation $\mathrm{T}$ and other numerical integration characteristics are the same as for $(\delta, Q)$ parametric plane, $\Delta \Omega=7.91667 \times 10^{-3}, 5.83333 \times 10^{-6}$. Numerical experiments show that for the larger hysteretic dissipation the chaotic regions areas are increased.

Figure 5 shows the phase portrait (a), hysteretic loop (b) and Poincaré map (e) of chaotic motion of the rotor. Parameters of motion correspond to the parameters of chaotic region depicted in Fig. 4 (b). The phase portrait (c), hysteretic loop (d) and Poincaré map (f) of the periodic rotor motion are also agree well with the obtained regions of regular/irregular behaviour of the rotor depicted in Fig. 4 (b). The influence of the magnetic control parameters $\gamma_{m}, \lambda_{m}$ on chaos occurring in the rotor vibrations can be observed in Fig. 6. The $\left(\gamma_{m}, Q\right)$ (a) and $\left(\lambda_{m}, Q\right)$ (b) parametric planes were uniformly sampled by $120 \times 120$ nodal points in the rectangles $\left(0<\gamma_{m} \leq 0.09 ; 0.00165<Q \leq 0.0019\right)$, $\Delta \gamma_{m}=7.5 \times 10^{-4}, \Delta Q=2.08333 \times 10^{-6} ;\left(450<\lambda_{m} \leq 630 ; 0.00145<Q \leq 0.0025\right), \Delta \lambda_{m}=1.5, \Delta Q=8.75 \times 10^{-6}$. The influence of the dynamic oil-film action characteristics on chaos occurring in the rotor motion can be observed in Fig. 7. One can see the restraining of chaotic regions with 
decreasing of hysteretic dissipation $(1-\delta)$. The $(C, Q)$ parametric plane was uniformly sampled by $120 \times 120$ nodal points in the rectangles $(0<C \leq 1.5 ; 0.0015<Q \leq 0.0021)$, $\Delta C=0.0125, \Delta Q=5 \times 10^{-6}$ (a) and $(0<C \leq 1.5 ; 0.0015<Q \leq 0.00225), \Delta C=0.0125, \Delta Q=6.25 \times 10^{-6}$ (b). The time period for the simulation $\mathrm{T}$ and other numerical integration characteristics are the same as for $(\delta, Q)$ parametric plane.

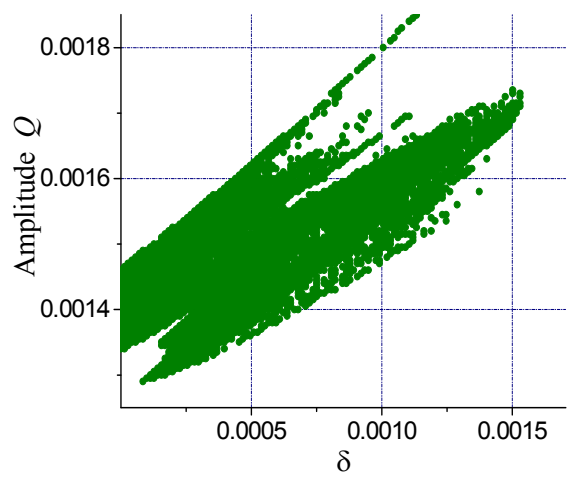

(a)

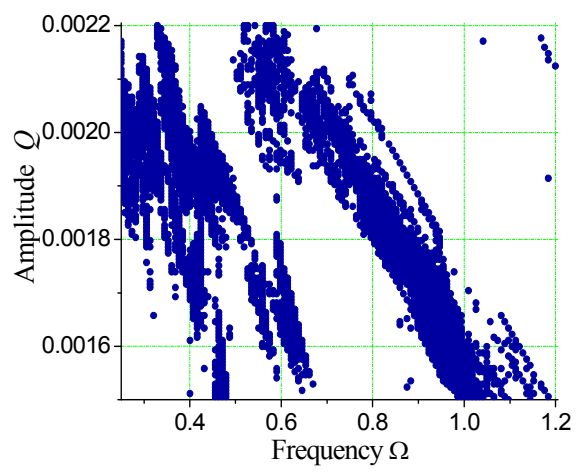

(b)

Fig. 4. (a) The influence of hysteretic dissipation parameter $\delta$ on chaos occurring in vertical vibrations of the rotor (28) in the case of rigid magnetic materials. The following parameters are fixed: $C=0.03, \gamma_{m}=0.001, \lambda_{m}=450, k_{z}=0.000055, \gamma=15, \beta=0.25, n=1.0, \Omega=0.87, Q_{0}=0, x_{0}=0$, $y_{0}=0, x(0)=y(0)=10^{-8}, \dot{x}(0)=\dot{y}(0)=0, z_{1}(0)=z_{2}(0)=0$;

(b) chaotic regions for the vertical vibrations of the rotor in the $(\Omega, Q)$ parametric plane with other parameters of the system fixed: $\delta=0.0001, C=0.2, \gamma_{m}=0, \lambda_{m}=500, k_{z}=0.000055, \gamma=15$, $\beta=0.25, n=1.0, Q_{0}=0, x_{0}=0, y_{0}=0, x(0)=y(0)=10^{-8}, \dot{x}(0)=\dot{y}(0)=0, \mathrm{z}_{1}(0)=\mathrm{z}_{2}(0)=0$. 


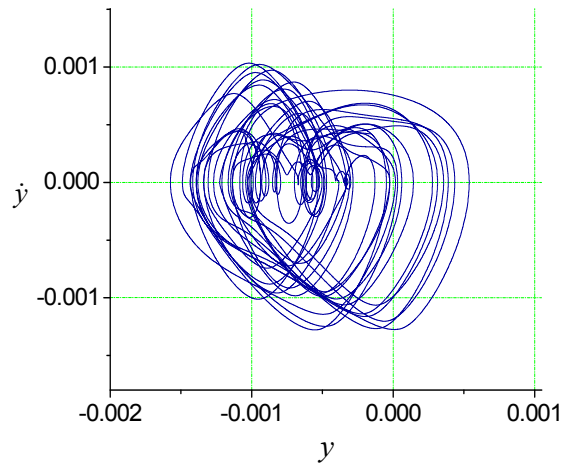

(a)

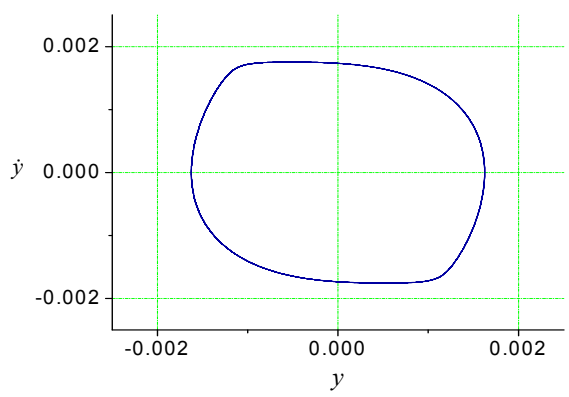

(c)

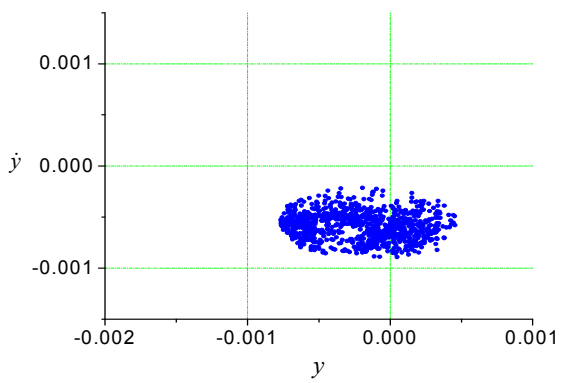

(e)

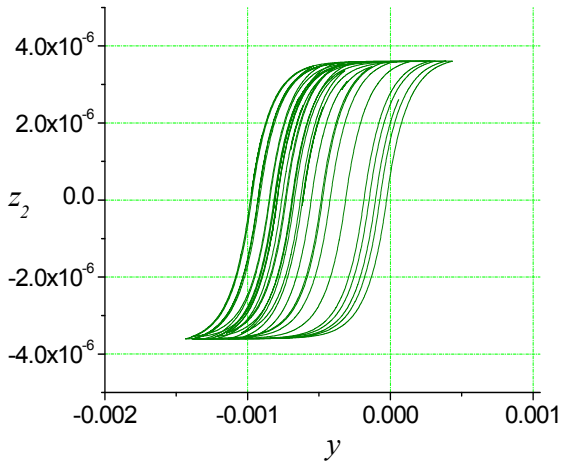

(b)

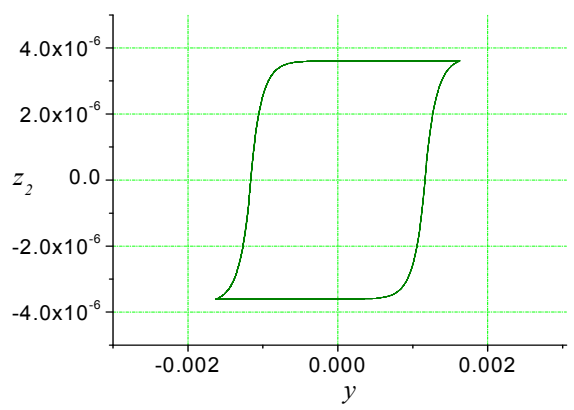

(d)

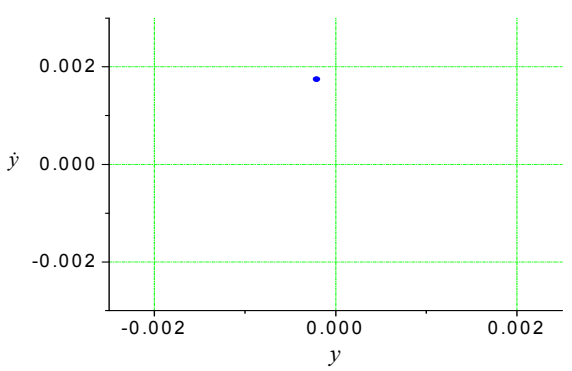

(f)

Fig. 5. Phase portraits (a), (c), hysteresis loops (b), (d) and Poincaré maps (e), (f) of the chaotic (a), (b), (e) and periodic (c), (d), (f) rotor motion that agree well with the chaotic/regular regions in Fig. 4 (b). The parameters $\delta=0.0001, C=0.2, \gamma_{m}=0, \lambda_{m}=500$, $k_{\mathrm{z}}=0.000055, \gamma=15, \beta=0.25, n=1.0, Q_{0}=0, x_{0}=0, y_{0}=0, x(0)=y(0)=10^{-8}, \dot{x}(0)=\dot{y}(0)=0$, $z_{1}(0)=z_{2}(0)=0$ are fixed; (a), (b), (e) $\Omega=0.87, Q=0.00177$; (c), (d), (f) $\Omega=1.2, Q=0.0017$ 


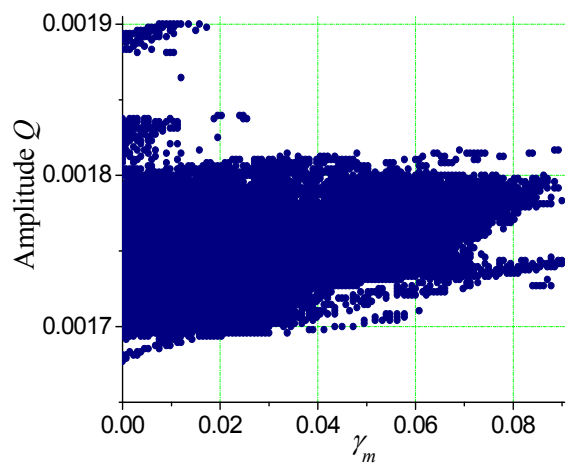

(a)

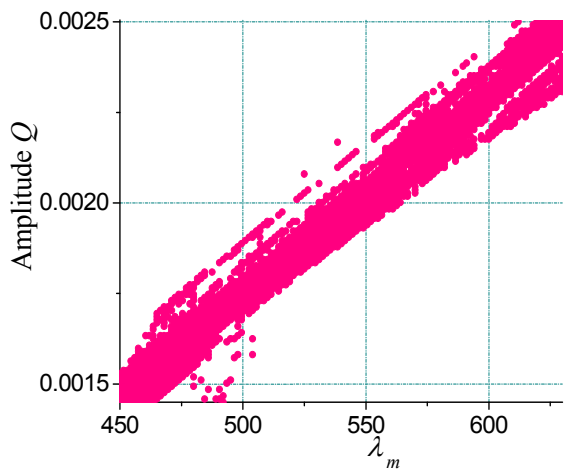

(b)

Fig. 6. The influence of the magnetic control parameters $\gamma_{m}$ (a) and $\lambda_{m}$ (b) on chaos occurring in vertical vibrations of the rotor (28) in the case of rigid magnetic materials. The parametric planes are depicted at (a) $\lambda_{m}=500$ and (b) $\gamma_{m}=0$ with other parameters of the system fixed: $\delta=0.000001, C=0.2, k_{z}=0.000055, \gamma=15, \beta=0.25, n=1.0, \Omega=0.87, Q_{0}=0, x_{0}=0, y_{0}=0$, $x(0)=y(0)=10^{-8}, \dot{x}(0)=\dot{y}(0)=0, z_{1}(0)=z_{2}(0)=0$

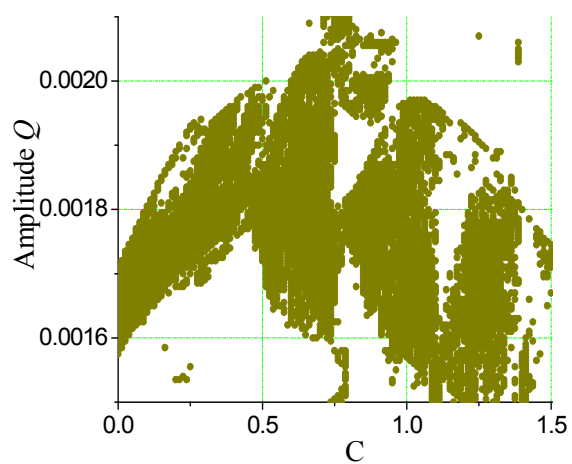

(a)

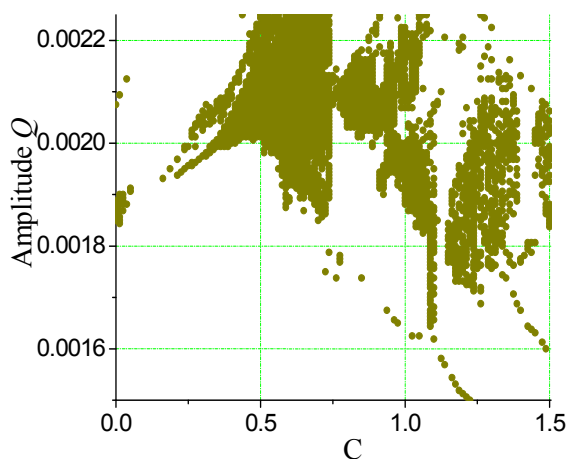

(b)

Fig. 7. The influence of the dynamic oil-film action characteristics on chaos occurring in vertical vibrations of the rotor (28) in the case of rigid magnetic materials. The parametric planes $(C, Q)$ are depicted at (a) $\delta=0.000001, \gamma_{m}=0$ and (b) $\delta=0.001, \gamma_{m}=0.03$ with other parameters of the system fixed: $\lambda_{m}=500, k_{z}=0.000055, \gamma=15, \beta=0.25, n=1.0, \Omega=0.87, Q_{0}=0, x_{0}=0$, $\mathrm{y}_{0}=0, x(0)=y(0)=10^{-8}, \dot{x}(0)=\dot{y}(0)=0, z_{1}(0)=z_{2}(0)=0$

In order to see if the rotor chaotic motion is accompanied by increasing of the amplitude of vibration, the amplitude level contours of the horizontal and vertical vibrations of the rotor have been obtained. In Fig. 8 (a) the amplitude level contours are presented in $\left(\gamma_{m}, Q\right)$ 
parametric plane with the same parameters as in Fig. 6 (a). Some "consonance" between the chaotic vibrations regions and the amplitude level contours is observed. At that the amplitudes of chaotic rotor vibrations are greater in comparison to the periodic vibrations. In Fig. $8(\mathrm{~b})$ the amplitude level contours are presented in $(C, Q)$ parametric plane with the same parameters as in Fig. 7 (a). Although some "consonance" between the chaotic regions of vibrations and the amplitude level contours is observed, it can not be concluded that chaos leads to essential increasing of the rotor vibrations amplitude.

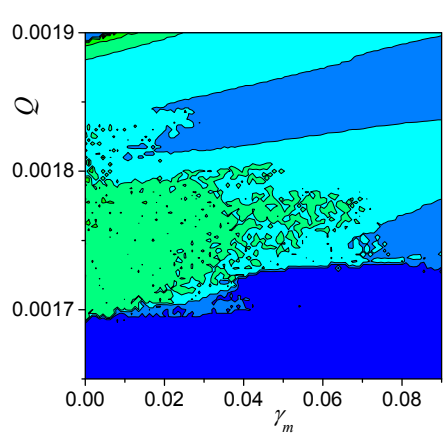

(a)

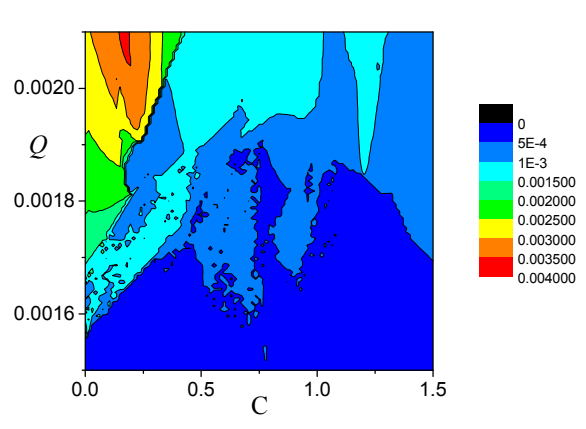

(b)

Fig. 8. The amplitude level contours of vertical vibrations of the rotor (28): (a) in the parametric plane $\left(\gamma_{m}, Q\right)$ that corresponds to Fig. $6(\mathrm{a})$; $(\mathrm{b})$ in the parametric plane $(C, Q)$ that corresponds to Fig. 7 (a)

\section{Conclusions}

2-dof non-linear dynamics of the rotor suspended in a magneto-hydrodynamic field is studied. In the case of soft magnetic materials the analytical solutions were obtained by means of the method of multiple scales. In the non-resonant case the system exhibits linear properties. The perturbation solutions are in good agreement with the numerical solutions. The cases of primary resonances with and without an internal resonance were investigated. The frequency-response curves were obtained. The saturation phenomenon was demonstrated. When the amplitude of the external excitation increases (or decreases), above some critical value the energy pumping between various submotions of the rotor occurs. A comparison of the analytical and numerical solutions based on the approximate harmonic analysis was made.

In the case of rigid magnetic materials, hysteresis was considered using the Bouc-Wen hysteretic model. Using the approach based on the analysis of the wandering trajectories the regions of chaotic vibrations of the rotor were found in various control parameter planes: amplitude of external harmonic excitation versus hysteretic dissipation, versus frequency of external harmonic excitation, dynamic oil-film action characteristics as well as versus the magnetic control parameters. The amplitude level contours of the horizontal and vertical vibrations of the rotor were obtained. Phase portraits and hysteretic loops are in good agreement with the chaotic regions obtained. Chaos was generated by hysteretic properties of the system considered. 


\section{References}

Awrejcewicz, J. \& Dzyubak, L. (2007). Hysteresis modelling and chaos prediction in oneand two-dof hysteretic models. Archive of Applied Mechanics, No.77, pp. 261-279

Awrejcewicz, J., Dzyubak, L. \& Grebogi, C. (2005). Estimation of chaotic and regular (stickslip and slip-slip) oscillations exhibited by coupled oscillators with dry friction. Non-linear Dynamics, No.42(2), pp. 383-394

Awrejcewicz, J. \& Mosdorf, R. (2003). Numerical Analysis of Some Problems of Chaotic Dynamics, WNT, Warsaw

Chang-Jian, C. W. \& Chen, C. K. (2009). Non-linear analysis of a rub-impact rotor supported by turbulent couple stress fluid film journal bearings under quadratic damping. Non-linear Dynamics, No.56, pp. 297-314

Dziedzic, K. \& Kurnik, W. (2002). Stability of a rotor with hybrid magneto-hydrodynamic support. Machine Dynamics Problems, No.26(4), pp. 33-43

Flores, P., Ambrosio, J., Claro, J. C. P., Lancarani, H. M. \& Koshy, C. S. (2009). Lubricated revolute joints in rigid multibody systems. Non-linear Dynamics, No.56, pp.277-295

Gasch, R., Nordmann, R. \& Pfützner, H. (2002). Rotordynamik, Springer, Berlin

Kurnik, W. (1995). Active magnetic antiwhirl control of a rigid rotor supported on hydrodynamic bearings. Machine Dynamics Problems, No.10, pp. 21-36

Li, J., Tian, Y., Zhang, W. \& Miao, S. F. (2006). Bifurcation of multiple limit cycles for a rotoractive magnetic bearings system with time-varying stiffness. International Journal of Bifurcation and Chaos

Muszyńska, A. (2005). Rotordynamics, CRC Press, Boca Raton

Nayfeh, A. H. \& Mook, D. T. (2004). Non-linear oscillations, Wiley, New York

Osinski, Z. (Ed.) (1998). Damping of Vibrations, A.A. Balkema, Rotterdam, Brookfield

Rao, J. S. (1991). Rotor Dynamics, Wiley, New York

Someya, T. (1998). Journal-Bearing Databook, Springer, Berlin

Tondl, A. (1965). Some Problems of Rotor Dynamics, Chapman \& Hall, London

Zhang, W. \& Zhan, X. P. (2005). Periodic and chaotic motions of a rotor-active magnetic bearing with quadratic and cubic terms and time-varying stiffness. Non-linear Dynamics, No.41, pp. 331-359 


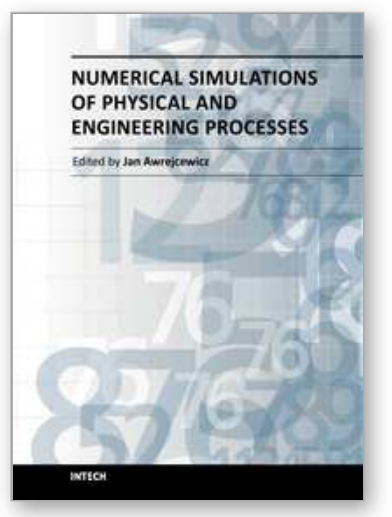

\author{
Numerical Simulations of Physical and Engineering Processes \\ Edited by Prof. Jan Awrejcewicz
}

ISBN 978-953-307-620-1

Hard cover, 594 pages

Publisher InTech

Published online 26, September, 2011

Published in print edition September, 2011

Numerical Simulations of Physical and Engineering Process is an edited book divided into two parts. Part I devoted to Physical Processes contains 14 chapters, whereas Part II titled Engineering Processes has 13 contributions. The book handles the recent research devoted to numerical simulations of physical and engineering systems. It can be treated as a bridge linking various numerical approaches of two closely interrelated branches of science, i.e. physics and engineering. Since the numerical simulations play a key role in both theoretical and application oriented research, professional reference books are highly needed by pure research scientists, applied mathematicians, engineers as well post-graduate students. In other words, it is expected that the book will serve as an effective tool in training the mentioned groups of researchers and beyond.

\title{
How to reference
}

In order to correctly reference this scholarly work, feel free to copy and paste the following:

Jan Awrejcewicz and Larisa P. Dzyubak (2011). Numerical Analysis of a Rotor Dynamics in the MagnetoHydrodynamic Field, Numerical Simulations of Physical and Engineering Processes, Prof. Jan Awrejcewicz (Ed.), ISBN: 978-953-307-620-1, InTech, Available from: http://www.intechopen.com/books/numericalsimulations-of-physical-and-engineering-processes/numerical-analysis-of-a-rotor-dynamics-in-the-magnetohydrodynamic-field

\section{INTECH}

open science | open minds

\author{
InTech Europe \\ University Campus STeP Ri \\ Slavka Krautzeka 83/A \\ 51000 Rijeka, Croatia \\ Phone: +385 (51) 770447 \\ Fax: +385 (51) 686166 \\ www.intechopen.com
}

\author{
InTech China \\ Unit 405, Office Block, Hotel Equatorial Shanghai \\ No.65, Yan An Road (West), Shanghai, 200040, China \\ 中国上海市延安西路65号上海国际贵都大饭店办公楼 405 单元 \\ Phone: +86-21-62489820 \\ Fax: $+86-21-62489821$
}


(C) 2011 The Author(s). Licensee IntechOpen. This chapter is distributed under the terms of the Creative Commons Attribution-NonCommercialShareAlike-3.0 License, which permits use, distribution and reproduction for non-commercial purposes, provided the original is properly cited and derivative works building on this content are distributed under the same license. 